School of Finance

University of St.Gallen

Electricity SPOT AND DeRIVATIVES PRICING WHEN MARKETS ARE INTERCONNECTED

ROLAND FÜSS

STEFFEN MAHRINGER

MARCEL PROKOPCZUK

WORKING PAPERS ON FinANCE No. 2013/23

SWISS INSTITUTE OF BANKING AND FINANCE (S/BF - HSG)

FIRST VERSION: SEPTEMBER 2013

THIS VERSION: JUNE 2015 


\title{
Electricity Spot and Derivatives Pricing when Markets are Interconnected
}

\author{
Roland Füss, ${ }^{*, \dagger}$ Steffen Mahringer, ${ }^{\ddagger}$ and Marcel Prokopczuk ${ }^{\S, q}$
}

This version: June 2015

\begin{abstract}
Increasing interconnectivity between electricity wholesale markets requires an efficient allocation scheme in order to provide access to scarce cross-border transmission capacities. In both the US and Europe, existing schemes have primarily induced economically inefficient interconnector use given that flows have to be nominated prior to spot market clearing. By contrast, the market coupling mechanisms recently rolled out in parts of Europe avoid these inefficiencies by implicitly allocating cross-border transmission capacity upon spot market clearance. In this paper, we show that these institutional aspects of market design clearly manifest in the empirical dynamics of both electricity spot and derivatives prices, and hence, do have important implications for pricing and hedging in these markets. Since traditional reduced-form models fail to reproduce such effects of market microstructure, we employ a fundamental multi-market model for electricity pricing in order to analyze how the key stylized facts of electricity prices are impacted by the different allocation schemes.
\end{abstract}

JEL classification: G12, G13, Q4, Q41

Keywords: Electricity Pricing, Fundamental Model, Multi-Market Modeling, Derivatives Pricing, Energy Market Coupling

\footnotetext{
${ }^{*}$ Swiss Institute of Banking and Finance (s/bf), University of St.Gallen, Rosenbergstr. 52, 9000 St.Gallen, Switzerland. Email: roland.fuess@unisg.ch

${ }^{\dagger}$ Centre for European Research (ZEW), Mannheim, Germany

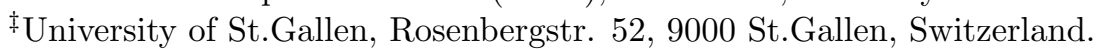

Email: steffen.mahringer@t-online.de

${ }^{\S}$ School of Economics and Management, Leibniz University Hannover, Königsworther Platz 1, 30167 Hannover, Germany. Email: prokopczuk@fmt.uni-hannover.de (corresponding author)

"We have benefited from the helpful comments of Michael Coulon, Karl Frauendorfer, Tarjei Kristiansen, and participants at the SIRE Conference on "Finance and Commodities" 2013, St.Andrews, the 55th Meeting of the EURO Working Group Commodities and Financial Modelling METU, Ankara, Turkey.
} 


\section{Introduction}

In the aftermath of market liberalization, energy markets in the US and Europe have been undergoing a number of significant structural developments and institutional changes that strongly affect the interplay of supply and demand, and hence, the general price formation process in these markets. In the case of electricity, regulatory developments, such as the introduction of emissions trading schemes, but also other aspects of market design, such as power exchanges admitting negative prices, have had a marked and long-lasting impact on the price dynamics of both spot and derivative contracts. Recently, in both US and Western European electricity markets, these evolutions have been overlaid with profound changes in the structure of the supply side that are primarily caused by a general, continued shift towards generation from renewable energy sources.

Since then, in view of rising shares of solar and wind capacities in many countries, the questions of (i) how to best integrate renewable generation into the networks and (ii) how to mitigate challenges relating to its intermittency and overall variability have ranked high on the agenda of system operators, regulatory authorities and respective policymakers. However, given that electricity flows are governed by the physical laws of Kirchhoff and Ohm rather than by the boundaries of geographic markets or otherwise defined price zones, the issue of integrating renewable generation should not be dealt with on a national level only, but instead is intimately related to the interconnectivity of adjacent electricity markets throughout Europe or the US as a whole. Hence, when assessing future target levels for generation from renewables, ${ }^{1}$ it is important to see that these are inherently tied to another aim, i.e., to enable a reliable and efficient transfer of "green" electricity across well-integrated markets and regions in the first place.

Within the EU, reaching this aim coincides with the intended establishment of the "Internal Electricity Market" (IEM), an initiative to support further integration of European electricity markets, with the ultimate goal of achieving full electricity price convergence across member states. ${ }^{2}$ Fostering integration between national markets, in

\footnotetext{
${ }^{1}$ For instance, the European Union (EU) targets a $20 \%$ share for renewable energy sources within its energy mix by the year 2020 .

${ }^{2}$ See, e.g., Article 60(2) of Directive 2009/72/EC: “(...) leading, in the long term, to price convergence."
} 
turn, requires substantial investments into transmission infrastructure both within and across national markets. Already in March 2002, the Barcelona European Council agreed on a minimum level of interconnectivity between member states of $10 \%$ of installed generation capacities within the respective markets. In this context, the European Network of Transmission System Operators for Electricity (ENTSO-E) estimates that for investment projects of "pan-European significance," a total capital expenditure of approx. EUR 104bn will be required until 2022 (ENTSO-E, 2012).

However, for the aim of creating a single electricity market across Europe, a well-defined and functioning market model of how to provide access to cross-border transmission capacity is at least as important as further investment into transmission infrastructure. Generally, only few interconnectors in Europe are uncongested $^{3}$ and over time, a variety of different congestion management methods had been developed to govern access to scarce transmission capacity for cross-border trade of electricity. In the past, these allocation mechanisms have primarily relied on explicit ex-ante schemes ${ }^{4}$, where traders first have to acquire transmission capacity in order to then arbitrage two interconnected, yet institutionally separated electricity markets. In Europe, in their most widespread form, explicit schemes are usually implemented as (sealed-bid) auction processes where capacity is allocated for different timeframes. ${ }^{5}$ While explicit ex-ante auctions of transmission capacity meet the requirement by the EU that access be provided based on "non-discriminatory market based solutions," ${ }^{6}$ they nevertheless lead to an inefficient market design: amongst a number of deficiencies, it is primarily the timing sequence of capacity and electricity spot markets that forces traders to acquire cross-border transmission capacity for a given direction before the spread in electricity spot prices between the two respective markets is actually determined. Hence, a trader's

\footnotetext{
${ }^{3}$ For instance, this is the case between Germany and Austria, where transmission capacity is sufficient and no auctioning of rights for interconnector use is required.

${ }^{4}$ See Füss et al. (2015) for an extensive discussion of explicit and implicit allocation schemes.

${ }^{5}$ Prior to the implementation of market coupling, explicit auction schemes were prevailing throughout Europe and were (or still are) used to allocate capacity for exchange between, e.g., England - France, France - Spain, France - Belgium, Belgium - The Netherlands, The Netherlands - Germany, or Germany - Denmark.

${ }^{6}$ See Regulations (EC) No. 1228/2003 and its follow-up No. 714/2009. Note that this rules out the use of other cross-border congestion management methods, such as rationing or allocation on a "first-come, first-served"-basis, given their lack of an inherent market-based mechanism.
} 
decision about both the direction and the amount of transmission capacity to be requested can only be based on an expectation of the spread, and in view of generally high levels of volatility in electricity spot markets, this noisy signal may often cause him to acquire capacity for the wrong direction.

Since these inefficiencies conflict with the objective of (day-ahead) price convergence within a single pan-European electricity market, the more efficient alternative of implicitly allocating cross-border capacity rights via market coupling is increasingly rolled out across European markets nowadays. Here, markets for transmission capacity and spot electricity are integrated and, hence, clear simultaneously, which allows for an optimal allocation of capacities and results in economically efficient cross-border flows. However, although the previous explicit ex-ante design has already been replaced by an implicit mechanism for a number of interconnectors, it will continue to play an important role wherever the harmonization efforts required for market coupling are too high or just infeasible. ${ }^{7}$

In the US, market architecture for most regions is fundamentally different from the uniform (zonal) pricing approach that is prevalent in Europe: instead, nodal pricing (or locational marginal pricing; LMP) has become the standard pricing approach. ${ }^{8}$ In an LMP-based market, the market area is subdivided into numerous pricing points, or nodes, for each of which an individual marginal electricity price is calculated. For instance, in the day-ahead market, participants submit bids and offers for specified point-to-point transactions which are then aggregated and matched by the central market administrator that clears the market. Thus, both day-ahead prices and corresponding flows are determined simultaneously and in the case of no congestion, marginal prices will be equal at each node. However, it is important to note that this approach optimally addresses transmission constraints within markets only, whereas the above mentioned problems in efficiently setting up cross-border transactions in Europe also apply for

\footnotetext{
${ }^{7}$ Also see, e.g., McInerney and Bunn (2013) on this argument. Moreover, should the market coupling algorithm (that determines the optimal cross-border flows) not be available due to technical problems, the explicit ex-ante scheme will be used as default option to allocate capacity.

${ }^{8}$ See, e.g., Bohn et al. (1984), Schweppe et al. (1988), and Hogan (1992) for an overview. Nodal pricing (in different forms) has been implemented, e.g., in California (CAISO), Texas (ERCOT), in the Midwest (MISO), New York (NYISO), and New England (ISO-NE) markets, in the PJM Interconnection, and in the Southwest Power Pool (SPP).
} 
transactions between markets in the US. For instance, if a trader wants to arbitrage the PJM and NYISO markets, his transaction still needs to be based on an expectation of the price spread between the two markets so that less-than-optimal or even adverse interconnector flows are a common problem on both sides of the Atlantic.

In the future, interconnectivity within the still fragmented US electricity grid is generally expected to further increase - e.g., through projects such as the planned "Tres Amigas Superstation," a bundle of three 5-gigawatt (GW) transmission lines that are supposed to unite the three regional grids in the US into a single national grid. ${ }^{9}$ While the market design for this emerging huge trading hub is still being worked out, a solution based on implicit allocation, i.e., market coupling, may likely be expected. ${ }^{10}$

In this paper, we show theoretically that the question of explicitly versus implicitly allocating cross-border transmission capacity induces important microstructure effects between interconnected electricity markets, ${ }^{11}$ which in turn has significant implications for pricing and hedging in these markets. Both the pace and scope of the above mentioned structural changes in electricity markets pose considerable challenges to market participants and, hence, require to use increasingly sophisticated models that are capable of adequately reproducing such changes in the "allocation regime." Moreover, since traditional reduced-form models for electricity pricing (although popular) are not capable of capturing these effects, model risk increases even further, leaving previously used pricing approaches unreliable in many instances.

As such, we contribute to the literature in the following ways: To the best of our knowledge, we are the first to analyze different set-ups for cross-border trade of electricity in a fully stochastic-dynamic setting, based on fundamental state variables such as demand

\footnotetext{
${ }^{9}$ Note that a grid or interconnection is defined as comprising several markets (such as PJM, NYISO, ISO-NE etc. for the Eastern Interconnection) that are electrically tied together and operate at a synchronized frequency. Currently, the three regional grids - the Eastern Interconnection, the Western Electricity Coordinating Council (WECC), and the Electric Reliability Council of Texas (ERCOT) - are only loosely tied together, which impedes, e.g., the transfer of "green" energy generated from wind in Texas or sun in Arizona to centers of high demand within the Eastern Interconnection.

${ }^{10}$ In 2012, Tres Amigas LLC and EPEX Spot have announced a joint cooperation agreement in order to "share mutual expertise in the development and coupling of their respective markets" (EPEX-Spot, 2012). EPEX Spot operates, amongst others, the coupled electricity spot markets in Germany and France.

${ }^{11}$ Unless otherwise stated, when using the term "explicit allocations," we always refer to explicit ex-ante allocations. See also Füss et al. (2015).
} 
and fuel prices for each market. ${ }^{12}$ More precisely, we propose a fundamental two-market model where, due to its granular structure, the influence of the above aspects of market design on the price formation mechanism can be mimicked and re-produced in a realistic way; thus, this setting not only addresses the shortcomings of reduced-form approaches, but also enables us to study in detail the interplay between the different "allocation regimes" and the ensuing dynamics for electricity spot and derivative prices. More generally, our framework allows for analytic pricing formulae for futures (and also options) under both allocation regimes, which adds to its general applicability and helps to retain tractability and ease of use for practitioners.

We further show how the most important stylized facts of electricity spot price dynamics - price spikes along with high levels of volatility - are altered through the introduction of market coupling in two adjacent markets. More precisely, our model both reproduces and allows to further investigate empirically observed facts such as a "volatility reduction effect" and a general softening of price spikes through market coupling. ${ }^{13}$ Taking a risk management perspective, we also show that interconnectivity of electricity markets can strongly impact the term structure of futures prices, such as reversing curves from backwardation into contango and vice versa.

Finally, the basic idea of directly reflecting key aspects of capacity allocation mechanisms in the price dynamics of the respective commodity can easily be transferred into a structural setting where gas pipeline capacities, storage access, or also bandwidth for data transfer have to be acquired prior to (or simultaneously with) commodity markets clearing. Hence, the general structure of our model can also serve as an important

\footnotetext{
${ }^{12}$ Note that while different ways of organizing cross-border trade of electricity have been thoroughly examined in the literature (see, e.g., Ehrenmann and Smeers, 2005), these analyses often rely on an exogenous deterministic and/or non-sequential setting.

${ }^{13}$ For instance, on 16 June 2013, given a combination of low demand and high levels of non-flexible generation, negative baseload prices were prevailing throughout the French, Belgian, and German day-ahead electricity markets. Since the surplus was particularly high in France, the market coupling mechanism did not achieve price convergence with neighbouring markets as flows required to equalize prices were exceeding interconnector capacities. However, as noted by EPEX Spot on that day, "(...) Market Coupling helped to absorb the price peaks despite the low price convergence" (EPEX-Spot, 2013).
} 
benchmark case for other network industries such as natural gas or telecommunications. ${ }^{14}$

The remainder of this paper is structured as follows: the next section develops the fundamental two-market model and shows how both explicit ex-ante and implicit allocation mechanisms for capacity rights can be accommodated within this framework. Numerical simulations and comparative-static analyses are employed to confirm that the model is capable to reproduce the key stylized facts of interconnected electricity markets. In Section III we derive futures pricing formulae and analyze the effect of interconnected electricity markets on their price dynamics. Section IV concludes.

\section{Modeling Two Interconnected Markets}

In order to conduct our theoretical analysis of the different market designs, we propose a fundamental two-market framework that is sufficiently granular to reflect the impact both of the most important underlying price drivers as well as of the different allocation mechanisms on price dynamics, while retaining flexibility and mathematical tractability at the same time. ${ }^{15}$ Taking the class of fundamental electricity pricing models as starting point ${ }^{16}$ we first adopt a setting similar to the one proposed in Füss et al. (2015a) in order to model electricity spot prices in each market. Similar to Skantze et al. (2004) and Coulon (2013), flows on the interconnector linking the two markets are then derived

\footnotetext{
${ }^{14}$ See, e.g., McDaniel (2003) and Stern and Turvey (2003) for further information and a general overview on capacity auctions in network industries. With respect to natural gas, note moreover that the general idea of electricity market coupling in Europe is currently planned to be transferred to the still fragmented gas markets where first coupling arrangements have recently been proposed (and partially implemented) by several European TSOs and gas exchanges. For instance, for the PEG Nord \& Sud zones in France (which are linked by a physical bottleneck), a gas market coupling project has been implemented in July 2011.

${ }^{15}$ From a technical point of view, it seems obvious that traditional reduced-form approaches are unsuited to include above aspects of market design into the price formation mechanism in a sufficiently detailed manner: irrespective of whether spot prices in two adjacent markets are modeled simultaneously in a bivariate reduced-form setting or whether the corresponding spot spread is modeled in a univariate setting, the ensuing price dynamics will not reflect the inherent interconnectivity between the markets and, hence, will always ignore the influence of the other market on domestic prices (or of both markets on the corresponding spread). Consequently, price dynamics will not be tied to any underlying economic causality that governs the exchange of cross-border flows, resulting in a general mis-specification of the model.

${ }^{16}$ See, e.g., Eydeland and Wolyniec (2002) for a general overview on the various approaches for electricity pricing, and Carmona and Coulon (2012) for a detailed introduction into the class of fundamental models for electricity pricing.
} 
endogenously, depending on whether corresponding cross-border transmission rights are assumed to be allocated explicitly ex-ante or implicitly via market coupling.

\section{A. Dynamics of Fundamental Variables}

In the following, we define a simplified continuous-time setting of two interconnected markets where electricity prices in each market $i=\{1,2\}$ are modeled as a function of underlying electricity demand $D_{i, t}$ as well as the cost of the marginal fuel $g_{i, t}$ used for electricity generation.

For the dynamics of electricity demand $D_{i, t}$ on a filtered probability space $\left(\Omega, \mathcal{F}^{D}, \mathbb{F}^{D}=\left(\mathcal{F}^{D}\right)_{t \in\left[0, T^{\star}\right]}, \mathbb{P}\right)$, a mean-reverting Gaussian Ornstein-Uhlenbeck (OU) process combined with a deterministic seasonal function has generally been considered an adequate modeling choice: ${ }^{17}$

$$
\begin{aligned}
D_{i, t} & =q_{i, t}+s_{D_{i}}(t), \\
\mathrm{d} q_{i, t} & =-\kappa_{q_{i}} q_{i, t} \mathrm{~d} t+\eta_{q_{i}} \mathrm{~d} B_{i, t},
\end{aligned}
$$

where $q_{i, t}$ is an OU-process for market $i$ with mean-reversion parameter $\kappa_{q_{i}}, B_{i, t}$ a standard Brownian motion, and $s_{D_{i}}(t)$ a deterministic seasonality function in order to capture the distinct seasonal patterns that electricity demand usually exhibits. ${ }^{18}$ Note that since we are modeling two geographically neighbouring markets, $q_{1, t}$ and $q_{2, t}$ are likely to be correlated, i.e., we allow for $\mathrm{d} B_{1, t} \mathrm{~d} B_{2, t}=\varrho_{q} \mathrm{~d} t$.

Regarding the type of fuel that is used for electricity generation, we only propose a general specification of the fuel price dynamics $g_{i, t}$ here since these will strongly depend on (i) the generation park of the respective electricity market to be modeled (e.g., coal- vs. gas-based markets) and (ii) potentially also on the maturity of the electricity (derivative)

\footnotetext{
${ }^{17}$ See, e.g., Aïd et al. (2009), Aïd et al. (2013), Carmona and Coulon (2012), or Füss et al. (2015a) for further reference.

${ }^{18}$ Commonly employed functional specifications include sine-functions or a combination of monthly dummy variables. See, e.g., Cartea and Villaplana (2008), Aïd et al. (2013), or Füss et al. (2015a).
} 
contract to be priced. ${ }^{19}$ Hence, on a filtered probability space $\left(\Omega, \mathcal{F}^{g}, \mathbb{F}^{g}=\left(\mathcal{F}^{g}\right)_{t \in\left[0, T^{\star}\right]}, \mathbb{P}\right)$, we assume prices for the marginal generating fuel $g_{i, t}$ to be governed by the commonly used mean-reverting one-factor model analyzed by Schwartz (1997):

$$
\begin{aligned}
\ln g_{i, t} & =X_{i, t}+s_{g_{i}}(t) \\
\mathrm{d} X_{i, t} & =-\kappa_{X_{i}} X_{i, t} \mathrm{~d} t+\eta_{X_{i}} \mathrm{~d} W_{i, t},
\end{aligned}
$$

where $X_{i, t}$ is an OU-process for market $i$ with mean-reversion parameter $\kappa_{X_{i}}, W_{i, t}$ a standard Brownian motion, and $s_{g_{i}}(t)$ a deterministic seasonality function. Again, we allow for potential correlation between the marginal fuel price processes in the two markets by setting $\mathrm{d} W_{1, t} \mathrm{~d} W_{2, t}=\varrho_{X} \mathrm{~d} t$. This also includes the special case that the marginal fuel used in both markets is identical, so that we can set $\varrho_{X}=1$ and $g_{1, t}=g_{2, t}$. Note that we assume zero correlation between demand and fuels both within each market as well as across markets, which not only helps to simplify valuation formulae but may also seem justified from an empirical point of view since (short-term) demand is generally inelastic with respect to fuel prices. ${ }^{20}$

Although more recent advances in the field of structural electricity price modeling ${ }^{21}$ have proposed to include additional state variables (such as available generation capacity $C_{i, t}$ ) or to allow for a model-endogenous determination of the merit order in multi-fuel set-ups, we confine the model to only include two state variables per market. Although our model could easily be extended to also include a capacity process $C_{i, t}$ or an additional fuel price process for each market, we refrain from doing so as this would neither change the general structure of the model nor extend the scope of our theoretical analysis, but rather

\footnotetext{
${ }^{19}$ In case of, e.g., long-term futures contracts, it may be necessary to use a model for generating fuels that adequately captures both short- and long-term fuel price dynamics, i.e., that needs to include two or more factors; see, e.g., Cartea and Williams (2008) for an overview in the case of natural gas. While we refrain from doing so, note that our one-factor setting for the dynamics of the underlying fuel price process could well be extended to also include log-normal multi-factor models for $g_{i, t}$.

${ }^{20}$ See, e.g., Pirrong and Jermakyan (2008).

${ }^{21}$ See, e.g., Aïd et al. (2013), Carmona et al. (2013), or Füss et al. (2015a).
} 
come at the cost of unnecessary complexity. ${ }^{22}$ As such, our stylized setting considers available domestic generation capacity as being fixed in both markets; alternatively, if a process for available capacity in each market shall nevertheless be integrated into the model, a straightforward approach would be to model excess capacity, i.e., reserve margins $M_{i, t}=C_{i, t}-D_{i, t}$, instead of alternatively treating $D_{i, t}$ and $C_{i, t}$ as separate (but possibly correlated) processes.

For the purpose of derivatives pricing further below, we need to recast our four-variate Gaussian setting under the risk-neutral measure $\mathbb{Q}$ by introducing (possibly time-varying) market prices of demand and fuel price risk, $\lambda_{q_{i}, t}$ and $\lambda_{X_{i}, t}$, respectively. However, given that, e.g., electricity demand $D_{i, t}$ is not a traded asset, this represents a non-hedgeable risk, which in turn yields an incomplete market setting. Consequently, "the" risk-neutral measure $\mathbb{Q}$ is no longer uniquely defined but instead comes along with an infinite number of alternative equivalent martingale measures. ${ }^{23}$ Hence, although straightforward to accommodate into our model, we choose to set $\lambda_{q_{i}, t}=\lambda_{X_{i}, t}=0$, implying $\mathbb{P}=\mathbb{Q}$. This does not affect the comparative-static analyses further below (which are insensitive to an exact specification of the market prices of demand and fuel price risk) but will rather help to simplify the following analysis.

To sum up, we obtain a four-variate Gaussian setting where conditional on time $t$,

\footnotetext{
${ }^{22}$ When empirically implementing our model for an electricity market where the marginal fuel may often change for some time of the day (e.g., coal during off-peak and natural gas during peak hours), a multi-fuel structural model might still appear more suitable at first glance. However, note that in electricity spot (day-ahead) markets, products are traded with respect to delivery of electricity during a certain hour of the day. As such, electricity contracts for delivery during night hours and contracts with delivery during, e.g., peak hours are essentially different commodities. Therefore, it is still possible to avoid implementing a multi-fuel setting by modeling electricity prices on an hourly basis (instead of modeling, e.g., daily (average) electricity prices) and merely fitting the price process $g_{i, t}$ to different fuel types during peak and off-peak hours.

${ }^{23}$ In case of an empirical implementation, $\lambda_{q_{i}, t}$ and $\lambda_{X_{i}, t}$ could be inferred by calibrating the model to observed prices of traded electricity contracts, as is done in Cartea and Villaplana (2008) or Füss et al. (2015a), for example. An alternative would be to set $\mathbb{Q}=\mathbb{Q}^{\text {min }}$, which denotes the minimal martingale measure proposed by Foellmer and Schweizer (1991). See, e.g., Aïd et al. (2009) for an application in the context of structural electricity price modeling.
} 
$q_{1, T}, q_{2, T}, X_{1, T}$, and $X_{2, T}$ are distributed as follows:

$$
\left[\begin{array}{c}
q_{1, T} \\
q_{2, T} \\
X_{1, T} \\
X_{2, T}
\end{array}\right] \sim N\left(\left[\begin{array}{l}
\mu_{q_{1}} \\
\mu_{q_{2}} \\
\mu_{X_{1}} \\
\mu_{X_{2}}
\end{array}\right],\left[\begin{array}{cccc}
\sigma_{q_{1}}^{2} & \rho_{q} \sigma_{q_{1}} \sigma_{q_{2}} & 0 & 0 \\
\rho_{q} \sigma_{q_{1}} \sigma_{q_{2}} & \sigma_{q_{2}}^{2} & 0 & 0 \\
0 & 0 & \sigma_{X_{1}}^{2} & \rho_{X} \sigma_{X_{1}} \sigma_{X_{2}} \\
0 & 0 & \rho_{X} \sigma_{X_{1}} \sigma_{X_{2}} & \sigma_{X_{2}}^{2}
\end{array}\right]\right),
$$

with

$$
\begin{aligned}
\mu_{q_{i}}(t, T) & =q_{i, t} \mathrm{e}^{-\kappa_{q_{i}}(T-t)}, \\
\sigma_{q_{i}}^{2}(t, T) & =\frac{\eta_{q_{i}}^{2}}{2 \kappa_{q_{i}}}\left(1-\mathrm{e}^{-2 \kappa_{q_{i}}(T-t)}\right), \\
\mu_{X_{i}}(t, T) & =X_{i, t} \mathrm{e}^{-\kappa_{X_{i}}(T-t)}, \\
\sigma_{X_{i}}^{2}(t, T) & =\frac{\eta_{X_{i}}^{2}}{2 \kappa_{X_{i}}}\left(1-\mathrm{e}^{-2 \kappa_{X_{i}}(T-t)}\right), \\
\rho_{q}(t, T) & =\frac{1}{\sigma_{q_{1}} \sigma_{q_{2}}} \frac{\varrho_{q} \eta_{q_{1}} \eta_{q_{2}}}{\kappa_{q_{1}}+\kappa_{q_{2}}}\left(1-\mathrm{e}^{-\left(\kappa_{q_{1}}+\kappa_{q_{2}}\right)(T-t)}\right), \\
\rho_{X}(t, T) & =\frac{1}{\sigma_{X_{1}} \sigma_{X_{2}}} \frac{\varrho_{X} \eta_{X_{1}} \eta_{X_{2}}}{\kappa_{X_{1}}+\kappa_{X_{2}}}\left(1-\mathrm{e}^{-\left(\kappa_{X_{1}}+\kappa_{X_{2}}\right)(T-t)}\right) .
\end{aligned}
$$

For ease of notation, $\mu_{(\cdot)}, \sigma_{(\cdot)}$, and $\rho_{(\cdot)}$ will always refer to $\mu_{(\cdot)}(t, T), \sigma_{(\cdot)}(t, T)$, and $\rho_{(\cdot)}(t, T)$ unless otherwise stated, e.g., as will be necessary when introducing our modeling setting for the case of an explicit ex-ante allocation of transmission rights.

\section{B. Spot Pricing Formulae}

In our setting, the two adjacent electricity markets shall be linked with each other by an interconnection line with capacity $K$. Electricity spot prices $P_{i, t}$ in market $i$ with $i=\{1,2\}$ are then defined as follows:

$$
\begin{aligned}
& P_{1, t}=\alpha_{1} g_{1, t}^{\delta_{1}} \exp \left(\beta_{1} D_{1, t}-\gamma_{1} J(t)\right), \\
& P_{2, t}=\alpha_{2} g_{2, t}^{\delta_{2}} \exp \left(\beta_{2} D_{2, t}+\gamma_{2} J(t)\right),
\end{aligned}
$$

where $D_{1, t}$ and $D_{2, t}$ represents completely inelastic electricity demand as given in Equations (1) and (2), and $J(t)$ is the flow of electricity on the interconnection line 
at time $t$. Note that as per standard economic reasoning, we assume that $\alpha_{i}>0, \beta_{i}>0$, $\gamma_{i}>0$, and $\delta_{i}>0$. Moreover, the market filtration is defined by $\mathcal{F}_{t}:=\mathcal{F}_{t}^{D} \vee \mathcal{F}_{t}^{g}$.

Regarding the above functional specification of the relationship between electricity prices $P_{i, t}$ and the state variables $D_{i, t}$ and $g_{i, t}$, the following needs to be taken into account: in Equations (6) and (7), electricity spot prices are derived based on an assumed relationship between generating fuels and demand, as is characteristic for the class of fundamental electricity pricing models (see, e.g., Skantze et al., 2000; Cartea and Villaplana, 2008; Pirrong and Jermakyan, 2008; Lyle and Elliott, 2009; or, more generally, Carmona and Coulon, 2012). These models bridge the gap between standard reduced-form settings on the one hand, and dynamic equilibrium models on the other hand, by combining aspects of both approaches: Although derived from an exogenously imposed functional specification rather than based on the optimization behavior of individual market participants, electricity prices $P_{i, t}$ are still derived in a ("reduced") equilibrium setting where supply equals demand. Using the exponential function to represent the characteristically highly convex curvature of the supply curve (merit-order curve) in electricity markets along with the assumption of completely inelastic demand, Equations (6) and (7) merely reflect the simple case of an equilibrium in two markets with inelastic demand and where imports (exports) are modeled as reductions (additions) to demand.

Also note that in Equations (6) and (7), the multiplicative structure of the RHS terms with respect to the generating fuels is clearly in accordance with the empirical observation that fuel prices are generally the main driver of merit-order or bid-stack dynamics in electricity markets (Eydeland and Geman, 1998; Pirrong and Jermakyan, 2008). ${ }^{24}$ However, when electricity prices are to be modeled on an hourly basis (as opposed to modeling daily average/baseload prices), occurrences of negative prices may be much more prominent, in which cases the above fuel price dependence breaks down. Given that in the event of a negative price spike, renewable generation bidding at negative prices tends to abound, state variables for coal or gas prices should not impact the negative

\footnotetext{
${ }^{24}$ Hence, the second part of our spot price formula, $\exp \left(\beta_{i} D_{i, t} \pm \gamma_{i} J(t)\right)$, is often interpreted as heat rate function that indicates how many units of generating fuels are required as inputs by generators to produce one unit of electricity.
} 
price dynamics (although there still may be a link to electricity demand $D_{i, t}$ ). While we do not focus on the issue of negative prices in order to keep the complexity of the following analysis at a tractable level, note that there are ways to adjust our model for that case. ${ }^{25}$

\section{Implicit vs. Explicit Allocation}

In the following, for the case when capacity rights are implicitly allocated (" $i a$;" "market coupling"), corresponding electricity spot prices will be denoted $P_{i, t}^{i a}$ as compared to $P_{i, t}^{e a}$ when the rights are explicitly allocated ("ea;" "connected, but non-coupled markets"). Note that while the two market regimes analyzed in this study share the same foundation, i.e., spot price model, they differ with respect to the determination of the interconnector flow $J$. Under either regime, $J$ will need to be determined based on a simplified allocation rule that leaves out complexities observed in reality, yet still yields a pricing formula that adequately reflects the key characteristics of market coupling (or explicit ex-ante allocation, respectively) in the ensuing price dynamics.

For the concept of market coupling, this allocation rule needs to mimic the economically optimal allocation of transmission capacities so that resulting cross-border flows are always directed from the lower- to the higher-priced market, just until any existing price differential between the two markets is exploited. Hence, we assume in a first step that by the joint effort of power exchanges, TSOs, and the market coupling office in the two markets, $J$ is set in order to reach perfect price convergence between the markets. The (unconstrained) flow $\widetilde{J}^{i a}(t)$ on the interconnector is hence derived as follows:

$$
\begin{aligned}
P_{1, t}^{i a} & \stackrel{!}{=} P_{2, t}^{i a} \\
\alpha_{1} g_{1, t}^{\delta_{1}} \exp \left(\beta_{1} D_{1, t}-\gamma_{1} \widetilde{J}^{i a}(t)\right) & =\alpha_{2} g_{2, t}^{\delta_{2}} \exp \left(\beta_{2} D_{2, t}+\gamma_{2} \widetilde{J}^{i a}(t)\right)
\end{aligned}
$$

\footnotetext{
${ }^{25}$ As a first "quick fix", dependence on fuel prices should be removed by setting $\delta_{i}=0$ so that prices could, by way of example, be defined as $P_{i, t}=-\alpha_{i} \mathrm{e}^{-\beta_{i} D_{i, t}}$, such as in Carmona et al. (2013). As a second step, this negative-price regime should be complemented with our standard positive-price regime so that electricity prices will then be determined in a classic regime-switching setting where state probabilities can additionally be dependent on electricity demand, so that prices would not be negative all the time, but only occasionally. See, e.g., Carmona and Coulon (2012) for an application in a one-market setting.
} 
Solving for $\widetilde{J}^{i a}(t)$ then yields:

$$
\widetilde{J}^{i a}(t)=\frac{1}{\gamma_{1}+\gamma_{2}}\left[\ln \alpha_{1}-\ln \alpha_{2}+\delta_{1} \ln g_{1, t}-\delta_{2} \ln g_{2, t}+\beta_{1} D_{1, t}-\beta_{2} D_{2, t}\right]
$$

By contrast, one of the key differences between implicit and explicit allocation of interconnector rights is the timing disconnect between when a trader is assigned transmission capacity and his physical flows need to be scheduled on the one hand, and when corresponding electricity (spot) markets clear on the other hand (see the illustration in Figure 1). More generally, also note that despite the differences between the respective institutional settings in Europe and the US (e.g., auction-based vs. non-auction based allocation), the explicit schemes are distinctly characterized by the above mentioned time lag which has been clearly identified as the main driver for the inherent economic inefficiency of these schemes. Hence, in order to derive interconnector flows for the explicit ex-ante regime, $\widetilde{J}^{e a}(t)$, the sequential nature of capacity and spot market clearance needs to be taken into account: in this case, the allocation of capacity rights is determined ex-ante at some point in time $\tau=t-k$ with $k$ fixed. Thus, at time $\tau$, when being allocated transmission capacity and scheduling flows for time $t=\tau+k$, market participants now base their decision on the expected price spread between $P_{1, \tau+k}$ and $P_{1, \tau+k}$, serving as best proxy in order to gauge the profitability of their intended cross-border transaction. Consequently, we shall assume that investors seek to schedule cross-border trades up until the (unconstrained) flow $\widetilde{J}^{\text {ea }}(t)$ suffices to set expected prices in the two markets equal:

$$
\begin{aligned}
\mathbb{E}_{t-k}\left[P_{1, t}^{e a}\right] & \stackrel{!}{=} \mathbb{E}_{t-k}\left[P_{2, t}^{e a}\right] \\
\mathbb{E}_{t-k}\left[\alpha_{1} g_{1, t}^{\delta_{1}} \exp \left(\beta_{1} D_{1, t}-\gamma_{1} \widetilde{J}^{e a}(t)\right)\right] & =\mathbb{E}_{t-k}\left[\alpha_{2} g_{2, t}^{\delta_{2}} \exp \left(\beta_{2} D_{2, t}+\gamma_{2} \widetilde{J}^{e a}(t)\right)\right]
\end{aligned}
$$

Solving the above for $\widetilde{J}^{e a}(t)$ yields:

$$
\begin{aligned}
\widetilde{J}^{e a}(t)= & \frac{1}{\gamma_{1}+\gamma_{2}}\left[\delta_{1} \mu_{X_{1}}(t-k, t)-\delta_{2} \mu_{X_{2}}(t-k, t)+\beta_{1} \mu_{q_{1}}(t-k, t)-\beta_{2} \mu_{q_{2}}(t-k, t)\right. \\
& +\frac{1}{2}\left(\delta_{1}^{2} \sigma_{X_{1}}^{2}(t-k, t)-\delta_{2}^{2} \sigma_{X_{2}}^{2}(t-k, t)\right)+\frac{1}{2}\left(\beta_{1}^{2} \sigma_{q_{1}}^{2}(t-k, t)-\beta_{2}^{2} \sigma_{q_{2}}^{2}(t-k, t)\right) \\
& \left.+\ln \alpha_{1}-\ln \alpha_{2}+\delta_{1} s_{g_{1}}(t)-\delta_{2} s_{g_{2}}(t)+\beta_{1} s_{D_{1}}(t)-\beta_{2} s_{D_{2}}(t)\right],
\end{aligned}
$$


where $\mu_{(\cdot)}(t-k, t)$ and $\sigma_{(\cdot)}^{2}(t-k, t)$ indicate the conditional expectation and variance relating to the respective processes at time $t$, yet taken at $t-k=\tau .{ }^{26}$

However, given that both $\widetilde{J}^{i a}(t)$ and $\widetilde{J}^{e a}(t)$, i.e., the optimal flows required to reach (expected) price convergence, may often surpass the actual capacity $K$ on the interconnector, the technically feasible flows $J^{i a}(t)$ and $J^{e a}(t)$ are both limited $(-K \leq$ $\left.J^{(\cdot)}(t) \leq K\right)$ and given as:

$$
J^{(\cdot)}(t)=\max \left(\min \left(\widetilde{J}^{(\cdot)}(t), K\right),-K\right)
$$

Hence, for either market regime, spot pricing formulae for the two markets need to take into account the above non-linearity in $J^{(\cdot)}(t)$ and have to distinguish three different scenarios. For markets 1 and 2 under market coupling, these are:

$$
\begin{aligned}
& P_{1, t}^{i a}=P_{1, t}^{i a, e x} \mathbb{I}_{\left\{\widetilde{J}^{i a}(t) \leq-K\right\}}+P_{1, t}^{i a, u n} \mathbb{I}_{\left\{-K<\widetilde{J}^{i a}(t)<K\right\}}+P_{1, t}^{i a, i m} \mathbb{I}_{\left\{\widetilde{J}^{i a}(t) \geq K\right\}}, \\
& P_{2, t}^{i a}=P_{2, t}^{i a, i m} \mathbb{I}_{\left\{\widetilde{J}^{i a}(t) \leq-K\right\}}+P_{2, t}^{i a, u n} \mathbb{I}_{\left\{-K<\widetilde{J}^{i a}(t)<K\right\}}+P_{2, t}^{i a, e x} \mathbb{I}_{\left\{\widetilde{J}^{i a}(t) \geq K\right\}},
\end{aligned}
$$

where $P_{1, t}^{i a, e x}\left(P_{2, t}^{i a, e x}\right)$ is the spot price at time $t$ in market 1 (market 2) if it is exporting electricity to market 2 (market 1 ). Physical interconnector flows are then constrained by the capacity of the transmission line and an amount of $K$ gigawatt (GW) units is exported from one market to the other. $P_{1, t}^{i a, \text { un }}\left(P_{2, t}^{i a, u n}\right)$ is the time- $t$ spot price if the interconnection line between the two markets is un-constrained. In such case, there is no congestion and market 1 (market 2) may either be exporting or importing at below the capacity limit $K$. Correspondingly, $P_{1, t}^{i a, i m}\left(P_{2, t}^{i a, i m}\right)$ is the spot price of electricity in market 1 (market 2 ) if it is in import-state and $J^{i a}(t)$ has reached its capacity limit. More explicitly, we have for

\footnotetext{
${ }^{26}$ For the explicit scheme, we indirectly assume that the allocation of capacity and the scheduling of physical flows coincide at time $\tau$, although for the example illustrated in Figure 1, scheduling of daily transactions is set after spot market clearance. However, note that the determination of $J^{e a}(t)$ based on expected price convergence does not consider the aspect of whether after the close of day-ahead markets, the trader will actually exercise his transmission right. For instance, if the right is out of the money, i.e., if the day-ahead spread turns out to be the opposite of his former belief at time $\tau=t-k$, the trader could close out his positions by resorting to the intraday platforms in both markets. Thus, the originally intended cross-border trade would be broken up into a domestic trade in each market, which, however, leaves unaffected the traders' commitments to buy/sell electricity in the day-ahead markets. Therefore, net demand $D_{i, t}-J^{e a}(t)$ in the importing market $\left(D_{j, t}+J^{e a}(t)\right.$ in the exporting market) would still be the same, as it is just another counterparty (i.e., the intraday market) to which electricity is delivered or from where it is supplied.
} 
market 1 (prices for market 2 are defined analogously):

$$
\begin{aligned}
& P_{1, t}^{i a, e x}=\alpha_{1} g_{1, t}^{\delta_{1}} \exp \left(\beta_{1} D_{1, t}-\gamma_{1}(-K)\right), \\
& P_{1, t}^{i a, \text { un }}=\alpha_{1} g_{1, t}^{\delta_{1}} \exp \left(\beta_{1} D_{1, t}-\gamma_{1} \widetilde{J}^{i a}(t)\right), \\
& P_{1, t}^{i a, i m}=\alpha_{1} g_{1, t}^{\delta_{1}} \exp \left(\beta_{1} D_{1, t}-\gamma_{1} K\right) .
\end{aligned}
$$

If market coupling is not in place and transmission rights are allocated explicitly instead, spot prices $P^{e a, e x}, P^{e a, u n}$, and $P^{e a, i m}$ are defined accordingly. ${ }^{27}$

Note, however, that our continuous-time setting generally implies that under the explicit ex-ante regime, the allocation procedure for transmission capacity and/or the related scheduling of transactions would be held during any infinitesimally small period of time, rather than, e.g., once a day in the case of day-ahead markets. Likewise, under market coupling, price convergence at any instance in time is (and will likely remain to be) out of technical reach. Hence, while adhering to a continuous-time framework for mathematical convenience, we follow the common assumption of interpreting electricity prices as discrete-time observations resulting from a price formation process which, in turn, is driven by the continuous-time dynamics of its underlying state variables. ${ }^{28}$ Similarly, in our setting, we can interpret $\widetilde{J}^{i a}(t)$ and $\widetilde{J}^{e a}(t)$ as hypothetical electricity flows which would prevail at any moment in time, yet which only materialize when it comes to price formation, i.e., when discretely observed at the scheduling date or at the time when establishing the market coupling flows.

\section{Analysis of Spot Prices}

In order to illustrate the mechanics of our spot pricing formulae, we employ a simulation with 1,000 time steps for each of the underlying fundamental factors $D_{1, t}, D_{2, t}, g_{1, t}$, and $g_{2, t}$. After simulating sample paths from the discretized processes for the state

\footnotetext{
${ }^{27}$ To conserve space, we do not state the corresponding differential equations for $P_{i, t}^{i a}$ and $P_{i, t}^{e a}$. Yet it is important to note that with an explicit ex-ante scheme, our spot price process is no longer Markovian given that interconnector flows $J^{e a}(t)$ were already determined by the values of our state variables at time $\tau=t-k$. The corresponding differential equation hence belongs to the class of stochastic delay differential equations (SDDE); see, e.g., Mohammed (1984) or Mao (1997) for further information.

${ }^{28}$ See, e.g., Benth et al. (2008) or Carmona and Coulon (2012) for a similar discussion.
} 
variables, we can then impose our different (exogenous) structural pricing relationships based on Equations (6) and (7), and depending on whether electricity prices $P_{1, t}^{(\cdot)}$ and $P_{2, t}^{(\cdot)}$ are to be derived under an explicit ex-ante or implicit (market coupling) allocation scheme. Comparability of the simulated spread time-series is ensured by using the same variates (and, hence, state variables) in each case, thus allowing us to analyze how shocks from underlying fundamental factors are reflected in electricity prices under the different regimes for cross-border trade. ${ }^{29}$ Results are shown in Figure 2 where in the top panel, we additionally have plotted the spread $P_{1, t}^{i s o}-P_{2, t}^{i s o}$ between two isolated electricity markets with no possibility of cross-border trading. The next two panels show the spreads $P_{1, t}^{e a}-P_{2, t}^{e a}$ and $P_{1, t}^{i a}-P_{2, t}^{i a}$, respectively. Finally, the two panels at the bottom present the corresponding endogenously determined interconnector flows that are related either to the spread under an explicit allocation or market coupling regime, respectively.

As can be seen in the top panel of Figure 2, without cross-border trade, the spread $P_{1, t}^{i s o}-P_{2, t}^{i s o}$ fluctuates widely around zero, reaching minimum and maximum values of approx. -22 EUR/MWh and $26 \mathrm{EUR} / \mathrm{MWh}$, respectively. As shown in the panel below, allowing for exchange between the markets under an explicit ex-ante scheme generally mitigates the price differential and thus improves price convergence. Taking as an example the characteristic spike of approx. $25 \mathrm{EUR} / \mathrm{MWh}$ in the top panel (marked red at simulation step 400), this spike is also reflected in the spread under the explicit ex-ante regime in the panel below, yet only at approximately half of its original magnitude (approx. $13 \mathrm{EUR} / \mathrm{MWh}$ ). At the same time, the spread series $P_{1, t}^{e a}-P_{2, t}^{e a}$ still looks slightly spikier as compared to the case of isolated markets, given that the generally smaller spread for explicit allocations (as compared to $P_{1, t}^{i s o}-P_{2, t}^{i s o}$ ) comes at the cost of inefficient interconnector flows that cause it to change its sign more frequently. However, if inefficient flows $J^{e a}(t)$ go in the wrong direction, spikes of the spot spread will even further increase

\footnotetext{
${ }^{29}$ More precisely, for the simulation, both markets are identically parametrized; the main input parameters to our model were set as follows: $s_{D_{1}}=s_{D_{2}}=40 \mathrm{GW}$ (i.e., to simplify, we refrain from incorporating seasonality for the time being), which compares against an interconnector capacity of $K=2 \mathrm{GW}$. Furthermore, we have set $\kappa_{q_{i}}=0.5, \kappa_{X_{i}}=0.001, \eta_{q_{i}}=1.0$, and $\eta_{X_{i}}=0.02$. These parameter values are in line with the empirical results of Füss et al. (2015a). Additionally, we assume $\varrho_{X}=\varrho_{q}=0.5$. We simulate on a daily basis so that having set $k=1$ implies that under explicit allocation, interconnector flows are determined one day ahead. For the more realistic case of $k<1$ (to reflect intraday timeframes), we can easily adjust our simulation, yet obtain the same qualitative results.
} 
as can be seen when examining the second spike highlighted in red towards the end of the sample period: in case of isolated markets, we have $P_{1, t}^{i s o}-P_{2, t}^{i s o}=14.15 \mathrm{EUR} / \mathrm{MWh}$, whereas $P_{1, t}^{e a}-P_{2, t}^{e a}$ increases to $16 \mathrm{EUR} / \mathrm{MWh}$ due to an adverse interconnector flow. Examining $J^{e a}(t)$ in the second to the last panel, we see that these adverse flows primarily occur when the fundamentally implied price differential between the two markets is about to reverse, e.g., as is indicated by the corresponding market coupling flows changing their direction from import to export (or vice versa). ${ }^{30}$

The "reflected" trajectory of the spread taking on either zero or positive (negative) values during the first (second) half of the sample period thereby merely reflects that in our simulation, spot electricity in market 1 is more expensive (cheaper) during most of that period; hence, a non-zero spread $P_{1, t}^{i a}-P_{2, t}^{i a}$ can always be observed whenever the price differential between the two markets is too high so that interconnector flows $J^{i a}(t)$ (that would be required to equalize prices in both markets) exceed the capacity limit $K$. This occurs in 219 out of 1000 simulation steps. Out of these 219 cases, in turn, it occurs that $J^{e a}(t)=J^{i a}(t)$ for 99 cases. In these cases, the fundamentals in the two markets are far apart so that even under an explicit allocation regime, full interconnector use is induced, and we have $P_{1, t}^{e a}-P_{2, t}^{e a}=P_{1, t}^{i a}-P_{2, t}^{i a}$ (see, e.g., first spike marked red at simulation step 400). In the remaining 120 cases with $\left|J^{i a}(t)\right|=K$, corresponding flows $J^{e a}(t)$ are inefficient so that spikes under market coupling can be further mitigated (see, e.g., second spike highlighted red).

Our model also allows to investigate more closely the empirically observed volatility reduction effect of market coupling discussed in Füss et al. (2015). In Figure 3, model sensitivities for the (unconditional) variance of log-spot returns of electricity prices under market coupling, $\ln \left(\frac{P_{i, t+1}^{i a}}{P_{i, t}^{i a}}\right)$, are provided and compared against the variance in case of

\footnotetext{
${ }^{30}$ Note that we strictly define only those interconnector flows $J^{e a}(t)$ as efficient where $J^{e a}(t)=J^{i a}(t)$. This implies that $J^{e a}(t)$ is only efficient when $J^{e a}(t)=J^{i a}(t)= \pm K$, which occurs in $10 \%$ of all cases. $76 \%$ of all derived flows $J^{e a}(t)$ are inefficient (yet go in the right direction), whereas the remaining $14 \%$ are adverse flows.
} 
isolated markets with no possibility of cross-border trade. ${ }^{31}$

In the LHS graph, we examine how higher variation in electricity prices (or, more precisely, in their underlying fundamental drivers) in market 1 is transmitted into market 2 , assuming a market coupling regime with interconnector capacity $K=\{0,2,4,12\}$. For that purpose, the ratio of instantaneous volatilities of the electricity demand processes, $\frac{\eta_{q_{1}}}{\eta_{q_{2}}}$, is varied over a range of 0.5 up to 2.5 (thereby keeping $\eta_{q_{2}}$ itself fixed). Consequently, for the case of isolated markets, the variance of log-spot returns in market 1 (dashed black line) is steeply increasing, thus merely reflecting the higher variance of the underlying state variable $D_{1, t}$ that feeds through to spot prices. Given that $K=0$, variance in market 2 remains unaffected, as indicated by the horizontal straight black line. Assuming a modest level of interconnectivity, $K=2$, the dashed dark grey line shows how the increase in variance for market 1 can be mitigated due to the optimal allocation of cross-border capacities under market coupling. The ensuing volatility reduction potential also manifests in the fact that for $\frac{\eta_{q_{1}}}{\eta_{q_{2}}}=1.0$, the two dark grey lines intersect at an approximate level of 0.006: thus, introducing market coupling between these two identically parametrized markets reduces return variance by some $25 \%$ alone. Compared to the status quo of isolated markets, for $K=2$, the increased demand volatility in market 1 only starts to affect market 2 for $\frac{\eta_{q_{1}}}{\eta_{q_{2}}}$ exceeding a ratio of approx. 2.1 , so that up to this threshold, any increase in $\eta_{q_{1}}$ is outweighed by the merits of coupling. Recalling the piecewise definition of spot prices (see Equations (12) and (13)), we see that spot prices in markets 1 and 2 will only differ in the import and export states. For higher levels of interconnectivity, the corresponding "weights" for these states, $\mathbb{I}_{\left\{\widetilde{J}^{i a}(t) \leq-K\right\}}$ and $\mathbb{I}_{\left\{\widetilde{J}^{i a}(t) \geq K\right\}}$, become smaller whereas $\mathbb{I}_{\left\{-K<\widetilde{J}^{i a}(t)<K\right\}}$ for the uncongested state increases, which causes the dynamics of the neighbouring market to increasingly feed through into the other market. This can also be illustrated technically, when inserting $\widetilde{J}^{i a}(t)$ from

\footnotetext{
${ }^{31}$ For the sensitivity analyses, we use the same parameters as in the simulation study. Also, we have set $\beta_{i}=0.1$ and $\delta_{i}=0.5$. For the LHS graph, we still assume state variables to be uncorrelated, $\varrho_{q}=\varrho_{X}=0$, although correlation obviously only impacts return variance in the two markets for $K>0$. For $K=0$, the unconditional variance of log-spot returns can then be computed as $2 \times 0.1^{2} \times 1^{2} \times(1-0.6065)+2 \times$ $0.5^{2} \times 0.2^{2} \times(1-0.9990)=0.0080$, as can be read off from both graphs.
} 
Equation (9) into $P_{1, t}^{i a, u n}$ from Equation (15):

$$
\begin{aligned}
P_{1, t}^{i a, \text { un }} & =\alpha_{1} g_{1, t}^{\delta_{1}} \exp \left(\beta_{1} D_{1, t}-\gamma_{1} \widetilde{J}^{i a}(t)\right) \\
& =\left(\alpha_{1} g_{1, t}^{\delta_{1}}\right)^{\frac{\gamma_{2}}{\gamma_{1}+\gamma_{2}}}\left(\alpha_{2} g_{2, t}^{\delta_{2}}\right)^{\frac{\gamma_{1}}{\gamma_{1}+\gamma_{2}}} \exp \left(\frac{\gamma_{2}}{\gamma_{1}+\gamma_{2}} \beta_{1} D_{1, t}+\frac{\gamma_{1}}{\gamma_{1}+\gamma_{2}} \beta_{2} D_{2, t}\right) .
\end{aligned}
$$

Consequently, spot prices effectively become a blend of all four state variables and converge towards each other, as do the light grey variance curves in the LHS graph of Figure 3. Finally, for high levels of interconnectivity, such as $K=12$, the resulting (perfect) price convergence between the two markets also equalizes return variances, as indicated by the red curves that virtually coincide (except for unrealistically high levels of $\left.\frac{\eta_{q_{1}}}{\eta_{q_{2}}}\right)$.

In the RHS graph of Figure 3, the variance of log-spot returns in market 1 is plotted against the correlation between state variables $\varrho_{q}$ or $\varrho_{X}$. Note that when varying $\varrho_{q}$, we keep $\varrho_{X}$ fixed at zero and vice versa. ${ }^{32}$ When examining both straight and dashed lines, we again see that higher levels of interconnector capacity $K$ generally allow for a higher volatility reduction potential as implied by our model. However, when varying $\varrho_{q}$, the upward-sloping straight lines imply that variance increases along with correlation, up until it reaches its upper bound, i.e., the case of isolated markets. ${ }^{33}$ Intuitively, this merely reflects the fact that arising synchronicity of demand shocks in the two markets makes it harder (or even impossible) for the market coupling mechanism to mitigate the resulting price spikes: if net supplies are scarce in both markets, even an economically optimal allocation of cross-border capacities cannot improve the situation. Technically, this is primarily due to the increasing contribution of the covariance between the demand processes $D_{i, t}$ to overall variance, as can be seen from $P_{1, t}^{i a, u n}$ in Equation (17). However,

\footnotetext{
${ }^{32}$ Note that the case of $\varrho_{q}>0$ and $\varrho_{X}>0$ should be more realistic from an empirical point of view. However, setting one of the correlation parameters to zero (while varying the other parameter) helps to better disentangle and relate the sensitivities to one or the other factor rather than having to consider mixing effects from both demand and fuel correlations being non-zero at the same time.

${ }^{33}$ Note, however, that although implied by our graph, this bound is not reached exactly. For $\varrho_{q}=1$, $\varrho_{X}=0$, and $K=12$, the difference between the variance of log-returns under market coupling and the variance in isolated markets will approximately amount to $\frac{1}{2} \delta_{i}^{2} \operatorname{Var}\left(\ln \frac{g_{i, t+1}}{g_{i, t}}\right)$, where we have assumed aforementioned identical parametrization of markets and where $\operatorname{Var}\left(\ln \frac{g_{i, t+1}}{g_{i, t}}\right)$ is the (unconditional) variance of log-returns from the fuel price process.
} 
there is an additional effect that becomes obvious when instead varying $\varrho_{X}$. Here, it is helpful to recall that the variance of log-returns is always also determined by the indicator functions $\mathbb{I}_{\{\cdot\}}$ that link the three scenarios in Equations (12) and (13). Their variance, in turn, is strongly linked to the overall variation in absolute-level interconnector flows $J^{i a}(t)$, as stated in Equation (9). Yet in contrast to the above, their variation is decreasing for higher levels of correlation $\varrho_{q}$ or $\varrho_{X}$, given that the state variables enter Equation (9) as pairwise differences. Hence, for a reasonable parametrization of our model, the term $\delta_{1} \ln g_{1, t}-\delta_{2} \ln g_{2, t}$ is the primary driver for the variation in $J^{i a}(t)$, which is large for $\varrho_{X}=-1$ and vanishes for $\varrho_{X}=1$. For modest levels of interconnectivity, this effect outweighs the "synchronicity" effect observed above and, consequently, causes the dashed lines in the RHS graph of Figure 3 to be downward sloping for $K=2$ and $K=4$. Finally, for $K=12$, it again suffices to focus on Equation (17) where we can see that for varying $\varrho_{X}$, the impact of increasing covariance between fuel prices on overall variance is disproportionately smaller than for varying $\varrho_{q}$, as is also reflected in the graph by the different slopes of the lines highlighted in red.

\section{Futures Pricing}

With the bulk of electricity trading generally taking place in liquid forward and futures markets, analyzing the effect of interconnected electricity markets on their price dynamics is at least as important as in the case of spot trading. Furthermore, given the prominent role of electricity forward and futures contracts for hedging purposes, the availability of closed-form pricing formulae for these contracts is particularly crucial from a risk management perspective.

Before starting to derive the pricing formula for a futures contract under both implicit and explicit allocation regimes, $F_{i, t}^{i a}(T)$ and $F_{i, t}^{e a}(T)$ with $i=\{1,2\}$ and maturity $T$, we state a useful result for calculating integrals over multivariate Gaussian densities: ${ }^{34}$

$$
\int_{-\infty}^{l} \mathrm{e}^{c x} \Phi\left(\frac{a+b x}{d}\right) \frac{\mathrm{e}^{-\frac{1}{2} x^{2}}}{\sqrt{2 \pi}} \mathrm{d} x=\mathrm{e}^{\frac{1}{2} c^{2}} \Phi_{2}\left(l-c, \frac{a+b c}{\sqrt{b^{2}+d^{2}}} ; \frac{-b}{\sqrt{b^{2}+d^{2}}}\right),
$$

\footnotetext{
${ }^{34}$ See, e.g., Carmona and Coulon (2012) for an application of this standard result in their multi-fuel structural electricity pricing model, but also Geske (1979) in the context of pricing compound options.
} 
where $a, b, c, d$, and $l$ are constants, $\Phi(\cdot)$ and $\Phi_{2}(\cdot, \cdot ; \rho)$ are the cumulative distribution functions of the univariate and bivariate (correlation $\rho$ ) standard normal distribution.

Based on the classic result that futures prices equal spot prices expected to prevail at maturity under the risk-neutral measure $\mathbb{Q}$ (recall our assumption of $\lambda_{q_{i}, t}=\lambda_{X_{i}, t}=0$ ), and using iterated conditioning, we can explicitly derive the time- $t$ futures price $F_{i, t}^{i a}(T)$ for market $i$ and maturity $T$ under market coupling:

$$
\begin{aligned}
F_{1, t}^{i a}(T)= & \mathbb{E}_{t}\left[P_{1, T}^{i a}\right] \\
= & \mathbb{E}_{t}\left[P_{1, T}^{i a, e x} \mathbb{I}_{\left\{\widetilde{J}^{i a}(T) \leq-K\right\}}+P_{1, T}^{i a, u n} \mathbb{I}_{\left\{-K<\widetilde{J}^{i a}(T)<K\right\}}+P_{1, T}^{i a, i m} \mathbb{I}_{\left\{\widetilde{J}^{i a}(T) \geq K\right\}}\right] \\
= & \int_{-\infty}^{\infty} \int_{-\infty}^{\infty} \int_{-\infty}^{\infty}\left(\int_{-\infty}^{l b^{i a}} \alpha_{1} g_{1, T}^{\delta_{1}} \exp \left(\beta_{1} D_{1, T}-\gamma_{1}(-K)\right) \phi\left(q_{1, T} \mid q_{2, T}\right) \mathrm{d} q_{1, T}\right. \\
& +\int_{l b^{i a}}^{u b^{i a}} \alpha_{1} g_{1, T}^{\delta_{1}} \exp \left(\beta_{1} D_{1, T}-\gamma_{1} \widetilde{J}^{i a}(T)\right) \phi\left(q_{1, T} \mid q_{2, T}\right) \mathrm{d} q_{1, T} \\
& \left.+\int_{u b^{i a}}^{\infty} \alpha_{1} g_{1, T}^{\delta_{1}} \exp \left(\beta_{1} D_{1, T}-\gamma_{1} K\right) \phi\left(q_{1, T} \mid q_{2, T}\right) \mathrm{d} q_{1, T}\right) \\
& \cdot \phi\left(q_{2, T}\right) \phi\left(X_{1, T} \mid X_{2, T}\right) \phi\left(X_{2, T}\right) \mathrm{d} q_{2, T} \mathrm{~d} X_{1, T} \mathrm{~d} X_{2, T},
\end{aligned}
$$

where $\phi\left(q_{1, T} \mid q_{2, T}\right)$ and $\phi\left(X_{1, T} \mid X_{2, T}\right)$ are the (risk-neutral) conditional densities of $q_{1, T}$ given $q_{2, T}$ and of $X_{1, T}$ given $X_{2, T}$, respectively. $\phi\left(q_{2, T}\right)$ and $\phi\left(X_{2, T}\right)$ are the (risk-neutral) unconditional densities of $q_{2, T}$ and $X_{2, T}$, respectively. The lower and upper bounds $l b^{i a}$ and $u b^{i a}$ for the innermost integrals over $q_{1, T}$ can be found by taking as starting point the corresponding inequations $\widetilde{J}^{i a}(T) \leq-K$ and $\widetilde{J}^{i a}(T) \geq K$, respectively (where $J^{i a}(T)$ is given by Equation (9)), and then solving for $q_{1, T}$. Based on the standard result for Gaussian densities stated above in Equation (18), and after some lines of algebra, we finally obtain the following closed-form solution for the futures price $F_{1, t}^{i a}(T):{ }^{35}$

$$
\begin{aligned}
F_{1, t}^{i a}(T)= & \mathbb{E}_{t}\left[P_{1, T}^{i a, e x}\right] \Phi\left(\frac{\mathcal{A}_{X}^{i a}+\mathcal{A}_{q}^{i a}-\underline{\mathcal{K}}}{\sqrt{\mathcal{C}_{X}^{i a}+\mathcal{C}_{q}^{i a}}}\right)+\mathbb{E}_{t}\left[P_{1, T}^{i a, i m}\right]\left[1-\Phi\left(\frac{\mathcal{A}_{X}^{i a}+\mathcal{A}_{q}^{i a}+\overline{\mathcal{K}}}{\sqrt{\mathcal{C}_{X}^{i a}+\mathcal{C}_{q}^{i a}}}\right)\right] \\
& +\mathbb{E}_{t}\left[P_{1, T}^{i a, u n}\right]\left[\Phi\left(\frac{\mathcal{B}_{X}^{i a}+\mathcal{B}_{q}^{i a}+\overline{\mathcal{K}}}{\sqrt{\mathcal{C}_{X}^{i a}+\mathcal{C}_{q}^{i a}}}\right)-\Phi\left(\frac{\mathcal{B}_{X}^{i a}+\mathcal{B}_{q}^{i a}-\underline{\mathcal{K}}}{\sqrt{\mathcal{C}_{X}^{i a}+\mathcal{C}_{q}^{i a}}}\right)\right]
\end{aligned}
$$

\footnotetext{
${ }^{35}$ Note that for $\mathbb{E}_{t}\left[P_{1, T}^{i a, e x}\right], \mathbb{E}_{t}\left[P_{1, T}^{i a, \text { un }}\right]$, and $\mathbb{E}_{t}\left[P_{1, T}^{i a, i m}\right]$, explicit expressions are stated in the technical Appendix $A$.
} 
with:

$$
\begin{aligned}
& \mathcal{A}_{X}^{i a}=\frac{\delta_{2}}{\beta_{1}} \mu_{X_{2}}-\frac{\delta_{1}}{\beta_{1}} \mu_{X_{1}}-\frac{\delta_{1}^{2}}{\beta_{1}} \sigma_{X_{1}}^{2}+\frac{\delta_{1} \delta_{2}}{\beta_{1}} \rho_{X} \sigma_{X_{1}} \sigma_{X_{2}}, \quad \mathcal{A}_{q}^{i a}=\frac{\beta_{2}}{\beta_{1}} \mu_{q_{2}}-\mu_{q_{1}}-\beta_{1} \sigma_{q_{1}}^{2}+\beta_{2} \rho_{q} \sigma_{q_{1}} \sigma_{q_{2}}, \\
& \mathcal{B}_{X}^{i a}=\frac{\delta_{2}}{\beta_{1}} \mu_{X_{2}}-\frac{\delta_{1}}{\beta_{1}} \mu_{X_{1}}-\frac{\delta_{1}}{\beta_{1}} \frac{\delta_{1} \gamma_{2}}{\gamma_{1}+\gamma_{2}} \sigma_{X_{1}}^{2}+\frac{\delta_{2}}{\beta_{1}} \frac{\delta_{2} \gamma_{1}}{\gamma_{1}+\gamma_{2}} \sigma_{X_{2}}^{2}+\frac{\left(\delta_{2} / \beta_{1}\right) \delta_{1} \gamma_{2}-\left(\delta_{1} / \beta_{1}\right) \delta_{2} \gamma_{1}}{\gamma_{1}+\gamma_{2}} \rho_{X} \sigma_{X_{1}} \sigma_{X_{2}}, \\
& \mathcal{B}_{q}^{i a}=\frac{\beta_{2}}{\beta_{1}} \mu_{q_{2}}-\mu_{q_{1}}-\frac{\beta_{1} \gamma_{2}}{\gamma_{1}+\gamma_{2}} \sigma_{q_{1}}^{2}+\frac{\beta_{2}}{\beta_{1}} \frac{\beta_{2} \gamma_{1}}{\gamma_{1}+\gamma_{2}} \sigma_{q_{2}}^{2}+\frac{\beta_{2} \gamma_{2}-\beta_{2} \gamma_{1}}{\gamma_{1}+\gamma_{2}} \rho_{q} \sigma_{q_{1}} \sigma_{q_{2}}, \\
& \mathcal{C}_{X}^{i a}=\left(\frac{\delta_{1}}{\beta_{1}}\right)^{2} \sigma_{X_{1}}^{2}-2 \frac{\delta_{1} \delta_{2}}{\beta_{1}^{2}} \rho_{X} \sigma_{X_{1}} \sigma_{X_{2}}+\left(\frac{\delta_{2}}{\beta_{1}}\right)^{2} \sigma_{X_{2}}^{2}, \quad \mathcal{C}_{q}^{i a}=\sigma_{q_{1}}^{2}-2 \frac{\beta_{2}}{\beta_{1}} \rho_{q} \sigma_{q_{1}} \sigma_{q_{2}}+\left(\frac{\beta_{2}}{\beta_{1}}\right)^{2} \sigma_{q_{2}}^{2}, \\
& \underline{\mathcal{K}}=\frac{\overline{\mathcal{K}}=\frac{\gamma_{1}+\gamma_{2}}{\beta_{1}} K-\frac{1}{\beta_{1}} \mathcal{S},}{\beta_{1}} K+\frac{1}{\beta_{1}} \mathcal{S}, \\
& \mathcal{S}=\ln \alpha_{1}-\ln \alpha_{2}+\delta_{1} s_{g_{1}}(T)-\delta_{2} s_{g_{2}}(T)+\beta_{1} s_{D_{1}}(T)-\beta_{2} s_{D_{2}}(T) .
\end{aligned}
$$

It is important to mention that the structure of the above formula merely reflects our 4-variate Gaussian setting where $F_{1, t}^{i a}(T)$ is simply expressed as an average of the respective futures contracts pertaining to each of the three states. The states are defined by $J^{i a}(T)$ and weighted by the probability of reaching each such state. ${ }^{36}$

If we are considering an explicit ex-ante allocation of capacity rights, it is again possible to derive analytic futures pricing formulae, even though our piecewise defined spot price process is no longer Markovian, because capacity and electricity spot markets do not clear simultaneously, as mentioned above. However, given that in our setting, the individual state variables $D_{i, t}$ and $g_{i, t}$ with $i=\{1,2\}$ are still Markovian, and since we only need to condition on a finite number of points in time of the past (i.e., on $\tau=T-k$ in this case), a closed-form expression for the futures price $F_{1, t}^{e a}(T)$ is still possible. Note first that, unlike for market coupling, $J^{e a}(T)$ was already determined at time $\tau=T-k$ and hence, is known at time $T$. At time $\tau$, and based on Equation (6), we then obtain:

$$
\begin{aligned}
F_{1, \tau}^{e a}(T)= & \mathbb{E}_{\tau}\left[P_{1, T}^{e a}\right] \\
= & \alpha_{1} \exp \left(\delta_{1}\left(\mu_{X_{1}}(\tau, T)+s_{g_{1}}(T)\right)+\beta_{1}\left(\mu_{q_{1}}(\tau, T)+s_{D_{1}}(T)\right)-\gamma_{1} J^{e a}(T)\right. \\
& \left.\quad+\frac{1}{2} \delta_{1}^{2} \sigma_{X_{1}}^{2}(\tau, T)+\frac{1}{2} \beta_{1}^{2} \sigma_{q_{1}}^{2}(\tau, T)\right) .
\end{aligned}
$$

The time- $t$ value of a futures contract under the explicit allocation regime with maturity

\footnotetext{
${ }^{36}$ However, note that in Equation (20), the factors involving the cumulative distribution function $\Phi(\cdot)$ do not exactly represent these probabilities given that for some state $z, \mathbb{E}\left[P_{1, T}^{i a, z} \mathbb{I}_{\{A\}}\right] \neq \mathbb{E}\left[P_{1, T}^{i a, z}\right] \mathbb{P}(A)$.
} 
$T, F_{1, t}^{e a}(T)$, can then be derived based on Equation (21). Using iterated conditioning, it follows:

$$
\begin{aligned}
F_{1, t}^{e a}(T)= & \mathbb{E}_{t}\left[\mathbb{E}_{\tau}\left[P_{1, T}^{e a}\right]\right] \\
=\mathbb{E}_{t}[ & \mathbb{E}_{\tau}\left[P_{1, T}^{e a, e x}\right] \mathbb{I}_{\left\{\widetilde{J}^{e a}(T) \leq-K\right\}}+\mathbb{E}_{\tau}\left[P_{1, T}^{e a, u n}\right] \mathbb{I}_{\left\{-K<\widetilde{J}^{e a}(T)<K\right\}} \\
& \left.\left.\quad+\mathbb{E}_{\tau}\left[P_{1, T}^{e a, i m}\right] \mathbb{I}_{\{\widetilde{J} e a}(T) \geq K\right\}\right]
\end{aligned}
$$

where $\widetilde{J}^{e a}(T)$ is $\mathcal{F}_{\tau}$-measurable. The explicit closed-form solution to the above equation is provided in the technical Appendix $B$.

Based on Equations (19) and (22), we can easily see that the futures price curves in both markets under either an explicit allocation or market coupling regime are actually a blend of the respective futures price curves for each state, i.e., $\mathbb{E}_{t}\left[P_{i, T}^{(\cdot), e x}\right], \mathbb{E}_{t}\left[P_{i, T}^{(\cdot), u n}\right]$, and $\mathbb{E}_{t}\left[P_{i, T}^{(\cdot), i m}\right]$ (as derived in the Appendix), each of which is essentially affine-linear in the underlying state variables (on log-basis). Revisiting our case of two identically parametrized markets, and assuming that the deseasonalized state variables have reverted back to their long-run means, i.e., $q_{i, t}=X_{i, t}=0$, we can now qualitatively argue that the futures price in an isolated market, $F_{i, t}^{i s o}(T)$, will always serve as an upper bound to its counterparts $F_{i, t}^{e a}(T)$ and $F_{i, t}^{i a}(T)$. Starting with $\varrho_{X}=\varrho_{q}=1$, we can verify from Equations (23) and (28) that $\mathbb{E}_{t}\left[P_{i, T}^{i a, \text { un }}\right]=\mathbb{E}_{t}\left[P_{i, T}^{\text {ea,un }}\right]=\mathbb{E}_{t}\left[P_{i, T}^{i s o}\right]$. In this case, given that the state variables will coincide in any instance, there will be no price differentials between the two markets that would provide an incentive for cross-border trade. Consequently, the weightings for the import and export states in Equations (20) and (25) must be zero, yielding $F_{i, t}^{i a}(T)=F_{i, t}^{e a}(T)=F_{i, t}^{i s o}(T)$. With decreasing correlations $\varrho_{X}$ and $\varrho_{q}$, two effects must be distinguished: first, $\mathbb{E}_{t}\left[P_{i, T}^{i a, u n}\right]$ and $\mathbb{E}_{t}\left[P_{i, T}^{e a, u n}\right]$ will always be smaller than $\mathbb{E}_{t}\left[P_{i, T}^{i s o}\right]$, which can again be seen from Equations (23) and (28) in Appendices A and B. Second, the above mentioned weightings for the import and export states will start to increase, and given the convexity of the exponential function, $\mathbb{E}_{t}\left[P_{i, T}^{(\cdot), e x}\right]$ will have a stronger effect on increasing futures prices than $\mathbb{E}_{t}\left[P_{i, T}^{(\cdot), i m}\right]$ on decreasing them. Note, however, that the corresponding weightings in Equations (20) and (25) are not symmetric but are adjusted by the terms $\underline{\mathcal{K}}$ and $\overline{\mathcal{K}}(\underline{\mathcal{L}}$ and $\overline{\mathcal{L}}$, respectively). As a consequence, the 
import weighting will always be higher than the export weighting, which - along with the first effect - will ensure that $F_{i, t}^{(\cdot)}(T) \leq F_{i, t}^{i s o}(T)$.

However, introducing cross-border trade between the two electricity markets may not only lead to a level shift in futures prices $F_{i, t}^{(\cdot)}(T)$ as compared to $F_{i, t}^{i s o}(T)$, but may also change the shape of the futures curve, as is shown in Figure 4. Here, we have assumed a higher price for the generating fuel in market 1 by setting $X_{1, t}=0.4$, whereas we retain $q_{i, t}=X_{2, t}=0$ and fix all other parameters as employed in Subsection D. Consequently, for the case of isolated markets, spot prices in market 1 will be higher than the futures prices that reflect the mean-reverting behavior of $X_{1, t}$ back to its zero-mean in the long run, which altogether results in a backwardated futures curve in market 1. Market 2, given that $K=0$, is not affected by the higher fuel prices in the adjacent market and has its futures curve in contango.

By contrast, with the possibility of cross-border exchange with either explicitly or implicitly allocated capacities, the curve in market 1 now is in contango for short-term maturities, which, in turn, forces the futures curve in market 2 into short-term backwardation. Hence, market 1 clearly benefits from indirectly accessing lower-cost generation in the neighboring market where prices now are correspondingly higher. Disaggregating further, the humped shape of the futures curve in market 1 can be explained by examining its individual components: with $X_{1, t}=0.4$ and for very short maturities, the probability of the more expensive market 1 nevertheless being in the export state is almost zero. The longer the time horizon, however, the more is $X_{1, t}$ expected to mean-revert so that the weighting for $\mathbb{E}_{t}\left[P_{1, T}^{(\cdot), e x}\right]$ (see Equations (20) and (25), respectively) increases. This effect dominates for maturities of up to slightly more than one year. Thereafter, it is outweighed by the facts that (i) both $\mathbb{E}_{t}\left[P_{1, T}^{(\cdot), e x}\right]$ and $\mathbb{E}_{t}\left[P_{1, T}^{(\cdot), i m}\right]$ are generally decreasing in $T$ (given our starting value for $X_{1, t}$ ) and that (ii) $\mathbb{E}_{t}\left[P_{1, T}^{(\cdot), \text { un }}\right]$ - although being approximately flat for any maturity - also exerts downward pressure on the "aggregate" futures curve since its associated weighting factor is decreasing for longer maturities.

Finally, it is interesting to see how the spread between futures prices will react to a change in the allocation mechanism, i.e., when switching from explicit allocations to 
market coupling (or vice versa). The bottom LHS graph of Figure 4 shows the spread between futures prices that corresponds to the top RHS and LHS graphs. As can be seen, switching from the explicit ex-ante mechanism to market coupling leads to a widening of the futures spread, which seems to be surprising given that spot price convergence under market coupling could be expected to also manifest in futures prices. The reason for this result is as follows: in order to assess how the futures spread will react to a change in the allocation regime, we need to compare Equations (19) and (22) for both markets. In view of the results from Equations (26) and (27) in Appendix B, and further simplifying, we shall now only focus on the differences between the indicator variables in the two expressions: in fact, it can be shown that for the more expensive market 1, a change to market coupling will always lead to a higher probability for the export state $\mathbb{I}_{\left\{\widetilde{J}^{i a}(T) \leq-K\right\}}$. To illustrate this fact, the bottom RHS graph of Figure 4 shows the distribution of interconnector flows $\widetilde{J}^{i a}(T)$ and $\widetilde{J}^{e a}(T)$ for an assumed maturity of $T=30$ days. From Equations (9) and (11), in turn, we know that $\widetilde{J}^{i a}(T)$ is a random variable (being itself a function of random variables) up until time $T$, whereas $\widetilde{J}^{e a}(T)$ will already be determined at time $T-k$. Consequently, the variance of the time- $T$ interconnector flows under market coupling will always be higher than for the case of explicit allocation, as is indicated by the fatter tails of the red distribution in the graph. More precisely, the area below the red curve for flows $\widetilde{J}^{i a}(T)<-K$ essentially reflects the probability of the more expensive market 1 being in the export state. Given our assumption of equal capacity limits in both directions of the interconnector, $\mathbb{P}\left(\widetilde{J}^{i a}(T)<\right.$ $-K$ ) for market 1 always increases as soon as the mean of flows is larger than zero. Hence, the weighting of the three different futures price components under market coupling will shift towards $\mathbb{E}_{t}\left[P_{1, T}^{i a, e x}\right]$ for market 1 and towards $\mathbb{E}_{t}\left[P_{2, T}^{i a, i m}\right]$ for market 2 . As such, roughly speaking, the more expensive market will even see slightly higher futures prices under market coupling, whereas the cheaper market can expect slightly lower futures prices, which altogether leads to a widening of the spread. ${ }^{37}$

\footnotetext{
${ }^{37}$ Note that our simplified reasoning leaves aside other effects such as (i) covariance terms between expectations and indicator functions or (ii) the fact that generally $\mathbb{E}_{t}\left[P_{1, T}^{i a, \text { un }}\right] \leq \mathbb{E}_{t}\left[P_{1, T}^{e a, \text { un }}\right]$, all of which are second-order effects, however, that do not outweigh the indicated directions of how futures prices move when changing from explicit to implicit allocations.
} 
However, when comparing these theoretical effects with empirical observations, the following caveat applies: in contrast to what is implied by our model, futures prices may nevertheless converge in reality given that actually available interconnector capacities $K$ under market coupling can be higher than the capacities that are available for the same line under an explicit scheme. In fact, there are two reasons for this. On the one hand, the issue of opposing flow nominations is treated differently under the two regimes; under an explicit scheme, such as explicit ex-ante auctions, transmission rights are allocated separately for each direction and flows nominated in opposite directions cannot be netted at all timescales. ${ }^{38}$

On the other hand, as is analyzed in Füss et al. (2015b) for the French-German border, the share of explicitly allocated yearly and monthly PTRs that are actually exercised by traders to ship electricity across borders has significantly declined since the introduction of market coupling. In this case, the UIOSI principle leads to a perfect hedging of the spread risk between two markets on a day-ahead basis, which causes market participants to increasingly opt for the financial compensation in case of no-exercise, rather than to set up a physical transaction in order to arbitrage the two markets by themselves.

Hence, these non-nominated capacities become available to the market coupling facilitator on a day-ahead basis, thus increasing $K$ under market coupling. As can be seen in the bottom LHS graph of Figure 4, assuming, by way of example, that the above arguments lead to a $25 \%$ increase in actually available interconnector capacity $K$ under market coupling now clearly leads to a lower futures spread as compared to the case of an explicit ex-ante regime.

\section{Conclusion}

Pricing and hedging in electricity markets has traditionally been a challenging and complex field. The complexity is primarily driven by the general non-storability of this

\footnotetext{
${ }^{38}$ See Höffler and Wittmann (2007) for a detailed analysis of netting in interconnector auctions. With respect to our example represented by the timeline in Figure 1, opposing flows nominated by holders of yearly or monthly transmission rights can be netted prior to the day-ahead auction of transmission capacity. However, this is not possible on the day-ahead stage itself. For further information, also see Hobbs et al. (2005), Bunn and Zachmann (2010), or Pellini (2012).
} 
commodity, however, additionally complicated by institutional specificities such as the differentiation of hourly electricity products, futures contracts delivering electricity over a certain period rather than at a fixed point in time, longer-term delivery contracts with cascading upon maturity, or other exotic products such as swing options. More recently, the increasing interconnectivity between national markets has further added to these complexities.

As has been shown, different ways of organizing cross-border trade between interconnected markets can significantly alter empirical price dynamics and, hence, render previously used, widespread modeling approaches inapplicable. As such, aspects of market design do and will continue to be of key interest for both practitioners and researchers, especially given two important developments: first, within the foreseeable future, interconnection capacity between markets is projected to remain scarce. Hence, price differentials between markets will persist, and so will different ways to manage ensuing congested cross-border flows. Second, the CWE market coupling focused on in this study is accompanied by a series of other regional coupling initiatives throughout Europe, such as the South-Western Europe (SWE) coupling between France, Spain and Portugal, or the North-Western Europe (NWE) coupling between CWE and the Scandinavian/Baltic countries. Within the "Price Coupling of Regions" (PCR) initiative, these different coupling projects are planned to be integrated in order to finally reach the EU policy goal of a single Internal Electricity Market. By then, the IEM is projected to be the world's largest electricity market, surpassing other mature markets in both the US and Australia.

Responding to the need for more sophisticated, accurate pricing models in this context, the class of fundamentally driven electricity pricing models has proven to offer a framework that provides sufficient granularity to reflect aspects of market design, while at the same time retaining flexibility and tractability to allow for closed-form solutions for many types of derivative contracts. Specifically, the model proposed in this study does not focus on the different explicit and implicit allocation mechanisms for interconnector capacity per se, but rather tries to mimic the outcomes of these different schemes by focusing on the resulting endogenous cross-border flows under each regime. 
The derived model dynamics and pricing formulae do address the shortcomings of the classic reduced-form approach and provide a rich framework to analyze how the key stylized facts of electricity spot prices as well as the term structure of futures prices change when markets are interconnected.

A further extension of our model could be to include a third interconnected market into our setting. While this will increase the complexity of the resulting pricing formulae, the general approach will stay the same, yet will depend on whether the additional market will be linked to both or only one of the two other markets. However, note that it may still be valid to apply a two-market model even if the empirical coupling mechanism involves more than two markets. As a matter of simplification, it may be advisable to reduce the number of markets to be modeled separately by aggregating those neighboring markets with structurally similar patterns of demand, or similar structures within their generation parks; with only infrequent congestion at their borders, or those markets that are small compared to their neighbor (e.g., Belgium vs. France). For instance, the CWE coupling could be decomposed into the German market on the one hand, and all other markets that are part of the Trilateral coupling on the other hand.

Another avenue for further research could focus on empirically implementing the model to price PTRs under market coupling, e.g., for the German-French border. Released data show that auctions for this border incite high investor interest, and the participation of the trading arms of several investment banks may boost liquidity even further. 


\section{Appendix}

\section{A. Futures Prices for the Implicit Regime}

Expressions for $\mathbb{E}_{t}\left[P_{1, T}^{i a, e x}\right], \mathbb{E}_{t}\left[P_{1, T}^{i a, \text { un }}\right]$, and $\mathbb{E}_{t}\left[P_{1, T}^{i a, i m}\right]$ in Equation (20) can easily be derived based on our piecewise definition of electricity spot prices in Equations (14) to (16) and using the properties of the lognormal distribution:

$$
\begin{aligned}
\mathbb{E}_{t}\left[P_{1, T}^{i a, \text { ex }}\right]=\alpha_{1} \exp \left(\delta_{1}\left(\mu_{X_{1}}+s_{g_{1}}(T)\right)+\beta_{1}\left(\mu_{q_{1}}+s_{D_{1}}(T)\right)+\gamma_{1} K+\frac{1}{2} \delta_{1}^{2} \sigma_{X_{1}}^{2}+\frac{1}{2} \beta_{1}^{2} \sigma_{q_{1}}^{2}\right) \\
\mathbb{E}_{t}\left[P_{1, T}^{i a, \text { un }}\right]=\exp \left(\frac{\delta_{1} \gamma_{2}}{\gamma_{1}+\gamma_{2}}\left(\mu_{X_{1}}+s_{g_{1}}(T)\right)+\frac{\delta_{2} \gamma_{1}}{\gamma_{1}+\gamma_{2}}\left(\mu_{X_{2}}+s_{g_{2}}(T)\right)+\frac{\beta_{1} \gamma_{2}}{\gamma_{1}+\gamma_{2}}\left(\mu_{q_{1}}+s_{D_{1}}(T)\right)\right. \\
\quad+\frac{\beta_{2} \gamma_{1}}{\gamma_{1}+\gamma_{2}}\left(\mu_{q_{2}}+s_{D_{2}}(T)\right)+\frac{\gamma_{2}}{\gamma_{1}+\gamma_{2}} \ln \alpha_{1}+\frac{\gamma_{1}}{\gamma_{1}+\gamma_{2}} \ln \alpha_{2} \\
\quad+\frac{1}{2}\left(\frac{\delta_{1} \gamma_{2}}{\gamma_{1}+\gamma_{2}}\right)^{2} \sigma_{X_{1}}^{2}+\frac{1}{2}\left(\frac{\delta_{2} \gamma_{1}}{\gamma_{1}+\gamma_{2}}\right)^{2} \sigma_{X_{2}}^{2}+\frac{\delta_{1} \delta_{2} \gamma_{1} \gamma_{2}}{\left(\gamma_{1}+\gamma_{2}\right)^{2}} \sigma_{X_{1}} \sigma_{X_{2}} \rho_{X} \\
\left.\quad+\frac{1}{2}\left(\frac{\beta_{1} \gamma_{2}}{\gamma_{1} \gamma_{2}}\right)^{2} \sigma_{q_{1}}^{2}+\frac{1}{2}\left(\frac{\beta_{2} \gamma_{1}}{\gamma_{1}+\gamma_{2}}\right)^{2} \sigma_{q_{2}}^{2}+\frac{\beta_{1} \beta_{2} \gamma_{1} \gamma_{2}}{\left(\gamma_{1}+\gamma_{2}\right)^{2}} \sigma_{q_{1}} \sigma_{q_{2}} \rho_{q}\right), \\
\mathbb{E}_{t}\left[P_{1, T}^{i a, i m}\right]=\alpha_{1} \exp \left(\delta_{1}\left(\mu_{X_{1}}+s_{g_{1}}(T)\right)+\beta_{1}\left(\mu_{q_{1}}+s_{D_{1}}(T)\right)-\gamma_{1} K+\frac{1}{2} \delta_{1}^{2} \sigma_{X_{1}}^{2}+\frac{1}{2} \beta_{1}^{2} \sigma_{q_{1}}^{2}\right) .
\end{aligned}
$$

\section{B. Futures Prices for the Explicit Ex-Ante Regime}

In order to derive an analytic pricing formula for the time- $t$ futures price $F_{1, t}^{e a}(T)$ with maturity $T$ in market 1 under the case of explicit allocations, we re-state Equation (22):

$$
\begin{aligned}
& F_{1, t}^{e a}(T)=\int_{-\infty}^{\infty} \int_{-\infty}^{\infty} \int_{-\infty}^{\infty}\left(\int_{-\infty}^{l b^{e a}} \mathbb{E}_{\tau}\left[P_{1, T}^{e a, e x}\right] \phi\left(q_{1, \tau} \mid q_{2, \tau}\right) \mathrm{d} q_{1, \tau}\right. \\
&+\int_{l b^{e a}}^{u b^{e a}} \mathbb{E}_{\tau}\left[P_{1, T}^{e a, u n}\right] \phi\left(q_{1, \tau} \mid q_{2, \tau}\right) \mathrm{d} q_{1, \tau} \\
&\left.+\int_{u b^{e a}}^{\infty} \mathbb{E}_{\tau}\left[P_{1, T}^{e a, i m}\right] \phi\left(q_{1, \tau} \mid q_{2, \tau}\right) \mathrm{d} q_{1, \tau}\right) \\
& \cdot \phi\left(q_{2, \tau}\right) \phi\left(X_{1, \tau} \mid X_{2, \tau}\right) \phi\left(X_{2, \tau}\right) \mathrm{d} q_{2, \tau} \mathrm{d} X_{1, \tau} \mathrm{d} X_{2, \tau},
\end{aligned}
$$

where $\phi\left(q_{1, \tau} \mid q_{2, \tau}\right)$ and $\phi\left(X_{1, \tau} \mid X_{2, \tau}\right)$ are the (risk-neutral) conditional densities of $q_{1, \tau}$ given $q_{2, \tau}$ and of $X_{1, \tau}$ given $X_{2, \tau}$, respectively. $\phi\left(q_{2, \tau}\right)$ and $\phi\left(X_{2, \tau}\right)$ are the (risk-neutral) unconditional densities of $q_{2, \tau}$ and $X_{2, \tau}$, respectively. The lower and upper bounds $l b^{e a}$ and $u b^{e a}$ for the innermost integrals over $q_{1, \tau}$ can be found in the same way as for $l b^{i a}$ and $u b^{i a}$ in the case of market coupling, i.e., by taking as starting point the corresponding inequations 
$\widetilde{J}^{e a}(T) \leq-K$ and $\widetilde{J^{e a}}(T) \geq K$, respectively (where $J^{e a}(T)$ is given by Equation $(11)$ ), and then solving for $q_{1, \tau}$. To preserve space, we introduce the following shorthand notation:

$$
b_{i}=\mathrm{e}^{-\kappa_{q_{i}}(T-\tau)}=\mathrm{e}^{-\kappa_{q_{i}} k}, \quad d_{i}=\mathrm{e}^{-\kappa_{X_{i}}(T-\tau)}=\mathrm{e}^{-\kappa_{X_{i}} k}
$$

Simplifying according to Equation (18) and after few manipulations, we finally obtain:

$$
\begin{aligned}
F_{1, t}^{e a}(T)= & \mathbb{E}_{t}\left[\mathbb{E}_{\tau}\left[P_{1, T}^{e a, e x}\right]\right] \Phi\left(\frac{\mathcal{A}_{X}^{e a}+\mathcal{A}_{q}^{e a}-\mathcal{\mathcal { L }}}{\sqrt{\mathcal{C}_{X}^{e a}+\mathcal{C}_{q}^{e a}}}\right)+\mathbb{E}_{t}\left[\mathbb{E}_{\tau}\left[P_{1, T}^{e a, i m}\right]\right]\left[1-\Phi\left(\frac{\mathcal{A}_{X}^{e a}+\mathcal{A}_{q}^{e a}+\overline{\mathcal{L}}}{\sqrt{\mathcal{C}_{X}^{e a}+\mathcal{C}_{q}^{e a}}}\right)\right] \\
& +\mathbb{E}_{t}\left[\mathbb{E}_{\tau}\left[P_{1, T}^{e a, u n}\right]\right]\left[\Phi\left(\frac{\mathcal{B}_{X}^{e a}+\mathcal{B}_{q}^{e a}+\overline{\mathcal{L}}}{\sqrt{\mathcal{C}_{X}^{e a}+\mathcal{C}_{q}^{e a}}}\right)-\Phi\left(\frac{\mathcal{B}_{X}^{e a}+\mathcal{B}_{q}^{e a}-\mathcal{\mathcal { L }}}{\sqrt{\mathcal{C}_{X}^{e a}+\mathcal{C}_{q}^{e a}}}\right)\right]
\end{aligned}
$$

where we have:

$$
\begin{aligned}
\mathcal{A}_{X}^{e a}= & \frac{\delta_{2} d_{2}}{\beta_{1} b_{1}} \mu_{X_{2}}(t, \tau)-\frac{\delta_{1} d_{1}}{\beta_{1} b_{1}} \mu_{X_{1}}(t, \tau)-\frac{\left(\delta_{1} d_{1}\right)^{2}}{\beta_{1} b_{1}} \sigma_{X_{1}}^{2}(t, \tau)+\frac{\delta_{1} \delta_{2} d_{1} d_{2}}{\beta_{1} b_{1}} \rho_{X}(t, \tau) \sigma_{X_{1}}(t, \tau) \sigma_{X_{2}}(t, \tau), \\
\mathcal{A}_{q}^{e a}= & \frac{\beta_{2} b_{2}}{\beta_{1} b_{1}} \mu_{q_{2}}(t, \tau)-\mu_{q_{1}}(t, \tau)-\beta_{1} b_{1} \sigma_{q_{1}}^{2}(t, \tau)+\frac{\beta_{2} b_{2}}{\beta_{1} b_{1}} \beta_{1} b_{1} \rho_{q}(t, \tau) \sigma_{q_{1}}(t, \tau) \sigma_{q_{2}}(t, \tau), \\
\mathcal{B}_{X}^{e a}= & \frac{\delta_{2} d_{2}}{\beta_{1} b_{1}} \mu_{X_{2}}(t, \tau)-\frac{\delta_{1} d_{1}}{\beta_{1} b_{1}} \mu_{X_{1}}(t, \tau)-\frac{\left(\delta_{1} d_{1}\right)^{2} \gamma_{2}}{\left(\beta_{1} b_{1}\right)\left(\gamma_{1}+\gamma_{2}\right)} \sigma_{X_{1}}^{2}(t, \tau)+\frac{\left(\delta_{2} d_{2}\right) \gamma_{1}}{\left(\beta_{1} b_{1}\right)\left(\gamma_{1}+\gamma_{2}\right)} \sigma_{X_{2}}^{2}(t, \tau) \\
& +\frac{\delta_{1} \delta_{2} d_{1} d_{2}\left(\gamma_{2}-\gamma_{1}\right)}{\beta_{1} b_{1}\left(\gamma_{1}+\gamma_{2}\right)} \rho_{X}(t, \tau) \sigma_{X_{1}}(t, \tau) \sigma_{X_{2}}(t, \tau), \\
\mathcal{B}_{q}^{e a}= & \frac{\beta_{2} b_{2}}{\beta_{1} b_{1}} \mu_{q_{2}}(t, \tau)-\mu_{q_{1}}(t, \tau)-\frac{\beta_{1} b_{1} \gamma_{2}}{\gamma_{1}+\gamma_{2}} \sigma_{q_{1}}^{2}(t, \tau)+\frac{\left(\beta_{2} b_{2}\right)^{2} \gamma_{1}}{\beta_{1} b_{1}\left(\gamma_{1}+\gamma_{2}\right)} \sigma_{q_{2}}^{2}(t, \tau) \\
& +\frac{\beta_{2} b_{2}\left(\gamma_{2}-\gamma_{1}\right)}{\gamma_{1}+\gamma_{2}} \rho_{q}(t, \tau) \sigma_{q_{1}}(t, \tau) \sigma_{q_{2}}(t, \tau), \\
\mathcal{C}_{X}^{e a}= & \left(\frac{\delta_{1} d_{1}}{\beta_{1} b_{1}}\right)^{2} \sigma_{X_{1}}^{2}(t, \tau)-2 \frac{\delta_{1} \delta_{2} d_{1} d_{2}}{\left(\beta_{1} b_{1}\right)^{2}} \rho_{X}(t, \tau) \sigma_{X_{1}}(t, \tau) \sigma_{X_{2}}(t, \tau)+\left(\frac{\delta_{2} d_{2}}{\beta_{1} b_{1}}\right)^{2} \sigma_{X_{2}}^{2}(t, \tau), \\
\mathcal{C}_{q}^{e a}= & \sigma_{q_{1}}^{2}(t, \tau)-2 \frac{\beta_{2} b_{2}}{\beta_{1} b_{1}} \rho_{q}(t, \tau) \sigma_{q_{1}}(t, \tau) \sigma_{q_{2}}(t, \tau)+\left(\frac{\beta_{2} b_{2}}{\beta_{1} b_{1}}\right)^{2} \sigma_{q_{2}}^{2}(t, \tau), \\
\mathcal{L}= & \frac{\gamma_{1}+\gamma_{2}}{\beta_{1} b_{1}} K+\frac{1}{\beta_{1} b_{1}} \mathcal{T}, \\
\overline{\mathcal{L}}= & \frac{\gamma_{1}+\gamma_{2}}{\beta_{1} b_{1}} K-\frac{1}{\beta_{1} b_{1}} \mathcal{T}, \\
\mathcal{T}= & \mathcal{S}+\frac{1}{2}\left(\delta_{1}^{2} \sigma_{X_{1}}^{2}(\tau, T)-\delta_{2}^{2} \sigma_{X_{2}}^{2}(\tau, T)\right)+\frac{1}{2}\left(\beta_{1}^{2} \sigma_{q_{1}}^{2}(\tau, T)-\beta_{2}^{2} \sigma_{q_{2}}^{2}(\tau, T)\right) .
\end{aligned}
$$

Finally, regarding explicit expressions for $\mathbb{E}_{t}\left[\mathbb{E}_{\tau}\left[P_{1, T}^{e a, e x}\right]\right], \quad \mathbb{E}_{t}\left[\mathbb{E}_{\tau}\left[P_{1, T}^{e a, i m}\right]\right]$, and $\mathbb{E}_{t}\left[\mathbb{E}_{\tau}\left[P_{1, T}^{e a, u n}\right]\right]$, we can see that by using iterated conditioning, we yield the following result for the first two expectations: 


$$
\begin{aligned}
\mathbb{E}_{t}\left[\mathbb{E}_{\tau}\left[P_{1, T}^{e a, e x}\right]\right] & =\mathbb{E}_{t}\left[P_{1, T}^{i a, e x}\right] \\
\mathbb{E}_{t}\left[\mathbb{E}_{\tau}\left[P_{1, T}^{e a, i m}\right]\right] & =\mathbb{E}_{t}\left[P_{1, T}^{i a, i m}\right]
\end{aligned}
$$

For $\mathbb{E}_{t}\left[\mathbb{E}_{\tau}\left[P_{1, T}^{e a, u n}\right]\right]$, based on the properties of the lognormal distribution and using iterated conditioning, we obtain after few lines of algebra:

$$
\begin{aligned}
\mathbb{E}_{t}\left[\mathbb{E}_{\tau}\left[P_{1, T}^{e a, u n}\right]\right]=\exp ( & \frac{\delta_{1} \gamma_{2}}{\gamma_{1}+\gamma_{2}}\left(\mu_{X_{1}}+s_{g_{1}}(T)\right)+\frac{\delta_{2} \gamma_{1}}{\gamma_{1}+\gamma_{2}}\left(\mu_{X_{2}}+s_{g_{2}}(T)\right)+\frac{\beta_{1} \gamma_{2}}{\gamma_{1}+\gamma_{2}}\left(\mu_{q_{1}}+s_{D_{1}}(T)\right) \\
& +\frac{\beta_{2} \gamma_{1}}{\gamma_{1}+\gamma_{2}}\left(\mu_{q_{2}}+s_{D_{2}}(T)\right)+\frac{\gamma_{2}}{\gamma_{1}+\gamma_{2}} \ln \alpha_{1}+\frac{\gamma_{1}}{\gamma_{1}+\gamma_{2}} \ln \alpha_{2} \\
& +\frac{1}{2}\left(\frac{\delta_{1} \gamma_{2}}{\gamma_{1}+\gamma_{2}}\right)^{2} d_{1}^{2} \sigma_{X_{1}}^{2}(t, \tau)+\frac{1}{2} \frac{\gamma_{2}}{\gamma_{1}+\gamma_{2}} \delta_{1}^{2} \sigma_{X_{1}}^{2}(\tau, T) \\
& +\frac{1}{2}\left(\frac{\delta_{2} \gamma_{1}}{\gamma_{1}+\gamma_{2}}\right)^{2} d_{2}^{2} \sigma_{X_{2}}^{2}(t, \tau)+\frac{1}{2} \frac{\gamma_{1}}{\gamma_{1}+\gamma_{2}} \delta_{2}^{2} \sigma_{X_{2}}^{2}(\tau, T) \\
& +\frac{\delta_{1} \delta_{2} \gamma_{1} \gamma_{2}}{\left(\gamma_{1}+\gamma_{2}\right)^{2}} d_{1} d_{2} \sigma_{X_{1}}(t, \tau) \sigma_{X_{2}}(t, \tau) \rho_{X}(t, \tau) \\
& +\frac{1}{2}\left(\frac{\beta_{1} \gamma_{2}}{\gamma_{1}+\gamma_{2}}\right)^{2} b_{1}^{2} \sigma_{q_{1}}^{2}(t, \tau)+\frac{1}{2} \frac{\gamma_{2}}{\gamma_{1}+\gamma_{2}} \beta_{1}^{2} \sigma_{q_{1}}^{2}(\tau, T) \\
& +\frac{1}{2}\left(\frac{\beta_{2} \gamma_{1}}{\gamma_{1}+\gamma_{2}}\right)^{2} b_{2}^{2} \sigma_{q_{2}}^{2}(t, \tau)+\frac{1}{2} \frac{\gamma_{1}}{\gamma_{1}+\gamma_{2}} \beta_{2}^{2} \sigma_{q_{2}}^{2}(\tau, T) \\
& \left.+\frac{\beta_{1} \beta_{2} \gamma_{1} \gamma_{2}}{\left(\gamma_{1}+\gamma_{2}\right)^{2}} b_{1} b_{2} \sigma_{q_{1}}(t, \tau) \sigma_{q_{2}}(t, \tau) \rho_{q}(t, \tau)\right) .
\end{aligned}
$$




\section{References}

R. Aïd, L. Campi, A.N. Huu, and N. Touzi. A structural risk-neutral model of electricity prices. International Journal of Theoretical and Applied Finance, 12(7):925-947, 2009.

R. Aïd, L. Campi, and N. Langrené. A structural risk-neutral model for pricing and hedging power derivatives. Mathematical Finance, 23(3):387-438, 2013.

F.E. Benth, J. Šaltytė-Benth, and S. Koekebakker. Stochastic Modeling of Electricity and Related Markets. World Scientific, London, 2008.

R.E. Bohn, M.C. Caramanis, and F.C. Schweppe. Optimal pricing in electrical networks over space and time. RAND Journal of Economics, 15(3):360-376, 1984.

D.W. Bunn and G. Zachmann. Inefficient arbitrage in inter-regional electricity transmission. Journal of Regulatory Economics, 37(3):243-265, 2010.

R. Carmona and M. Coulon. A survey of commodity markets and structural model for electricity prices. In F.E. Benth, editor, Financial Engineering for Energy Asset Management and Hedging in Commodity Markets; Proceedings from the special thematic year at the Wolfgang Pauli Institute. Vienna, 2012.

R. Carmona, M. Coulon, and D. Schwarz. Electricity price modeling and asset valuation: A multi-fuel structural approach. Mathematics and Financial Economics, 7(2):167-202, 2013.

A. Cartea and P. Villaplana. Spot price modeling and the valuation of electricity forward contracts: The role of demand and capacity. Journal of Banking \& Finance, 32(12): 2502-2519, 2008.

A. Cartea and T. Williams. UK gas markets: The market price of risk and applications to multiple interruptible supply contracts. Energy Economics, 30(3):829-846, 2008.

M. Coulon. Forward Price Approximation for Coupled European Electricity Markets. Working Paper, University of Princeton, 2013.

A. Ehrenmann and Y. Smeers. Inefficiencies in European congestion management proposals. Utilities Policy, 13(2):135-152, 2005.

ENTSO-E. Ten-Year Network Development Plan. 2012. URL https://www .entsoe.eu/ major-projects/ten-year-network-development-plan/tyndp-2012. 
EPEX-Spot. Joint Cooperation Agreement Executed Between EPEX Spot And Tres Amigas. 2012. URL http://static.epexspot.com/document/17470/2012-01-10_ Tres\%20Amigas_EPEXSPOT.pdf.

EPEX-Spot. APX, BELPEX and EPEX Spot statement about negative baseload prices on 16 june 2013. 2013. URL http://www.epexspot.com/en/press-media/ news/details/news/APX_Belpex_and_EPEX_SPOT_statement_about_negative_ baseload_prices_on_16_June_2013.

A. Eydeland and H. Geman. Pricing Power Derivatives. RISK, 11:71-73, 1998.

A. Eydeland and K. Wolyniec. Energy and Power Risk Management: New Developments in Modeling, Pricing, and Hedging. John Wiley and Sons, New York, 2002.

H. Foellmer and M. Schweizer. Hedging of contingent claims under incomplete information. In M.H.A. Davis and R.J. Elliott, editors, "Applied Stochastic Analysis", Stochastics Monographs, Vol. 5. Gordon and Breach, London/New York, 1991.

R. Füss, S. Mahringer, and M. Prokopczuk. Electricity Derivatives Pricing with ForwardLooking Information. Journal of Economic Dynamics and Control, forthcoming, 2015a.

R. Füss, S. Mahringer, and M. Prokopczuk. Electricity market coupling and the pricing of transmission rights: An option-based approach. Working Paper, University of St.Gallen, 2015b.

R. Geske. The valuation of compound options. Journal of Financial Economics, 7:63-81, 1979.

F. Höffler and T. Wittmann. Netting of Capacity in Interconnector Auctions. The Energy Journal, 28(1):113-144, 2007.

B.F. Hobbs, A.M. Rijkers, and M.G. Boots. The More Cooperation, The More Competition? A Cournot Analysis of the Benefits of Electric Market Coupling. The Energy Journal, 26(4):69-97, 2005.

W.W. Hogan. Contract Networks for Electric Power Transmission. Journal of Regulatory Economics, 4(3):211-242, 1992.

M.R. Lyle and R.J. Elliott. A 'simple' hybrid model for power derivatives. Energy Economics, 31(5):757-767, 2009.

X. Mao. Stochastic Differential Equations and Applications. Horwood, Chichester, 1997. 
T. McDaniel. Auctioning access to networks: evidence and expectations. Utilities Policy, 11(1):33-38, 2003.

C. McInerney and D.W. Bunn. Valuation anomalies for interconnector transmission rights. Energy Policy, 55:565-578, 2013.

S.-E. A. Mohammed. Stochastic Functional Differential Equations. Research Notes in Mathematics 99. Pitman Books, London, 1984.

E. Pellini. Measuring the impact of market coupling on the Italian electricity market. Energy Policy, 48:322-333, 2012.

C. Pirrong and M. Jermakyan. The price of power: The valuation of power and weather derivatives. Journal of Banking \& Finance, 32(12):2520-2529, 2008.

RTE. Access Rules for Imports and Exports on the French Public Power Transmission System. 2009a. URL http://clients.rte-france.com/lang/an/clients_traders_ fournisseurs/services_clients/inter_contrats.jsp.

RTE. Rules for Capacity Allocation by Explicit Auctions within Central West Europe Region (CWE Auction Rules). 2009b. URL http://clients.rte-france. com/lang/an/clients_traders_fournisseurs/services_clients/initiatives_ regionales.jsp.

E.S. Schwartz. The stochastic behavior of commodity prices: Implications for valuation and hedging. Journal of Finance, 52(3):923-973, 1997.

F.C. Schweppe, M.C. Caramanis, R.D. Tabors, and R.E. Bohn. Spot Pricing of Electricity. Kluwer Academic Publishers, Boston, MA, 1988.

P. Skantze, A. Gubina, and M. Ilic. Bid-based Stochastic Model for Electricity Prices: The Impact of Fundamental Drivers on Market Dynamics. MIT Energy Laboratory Publication, 2000.

P. Skantze, M. Ilic, and A. Gubina. Modelling locational price spreads in competitive electricity markets; applications for transmission rights valuation and replication. IMA Journal of Management Mathematics, 15(4):291-319, 2004.

J. Stern and R. Turvey. Auctions of capacity in network industries. Utilities Policy, 11 (1):1-8, 2003. 


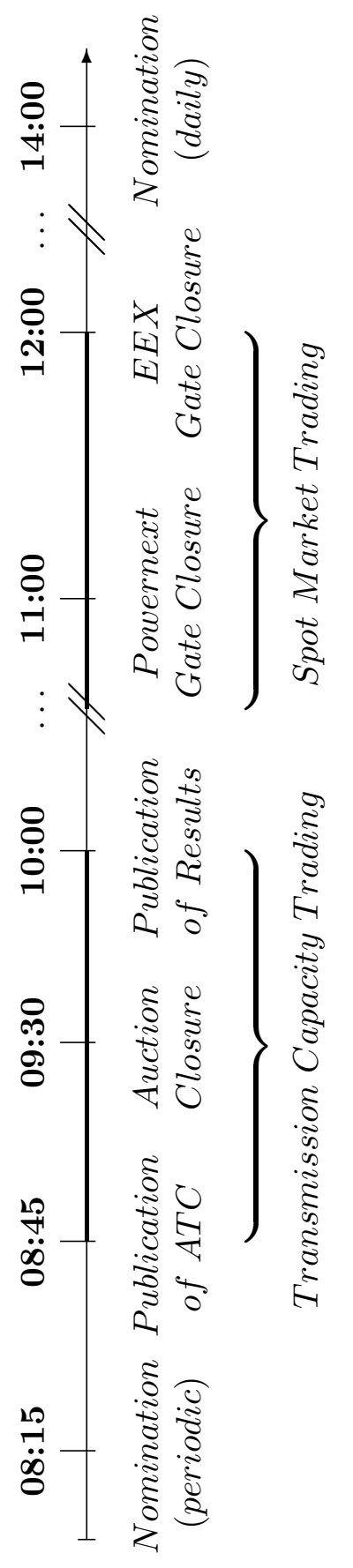

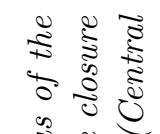

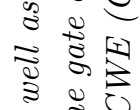

\& $\approx$

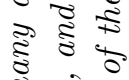

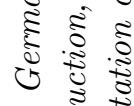

胥胥

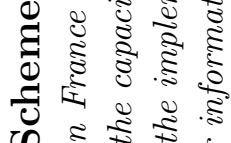

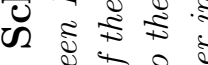

.․ำ

药

要

毒

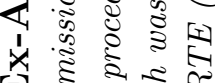

I है क ती

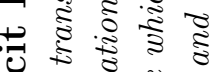

离. है है

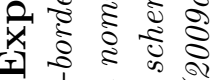

के

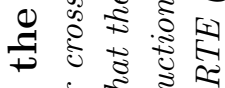

के ई5 స :

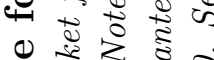

.尹 है है

द

स

-

कृ

20000

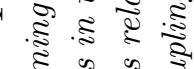

हิ

क है है

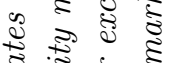

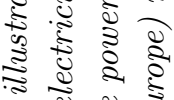

ข

先

స. 


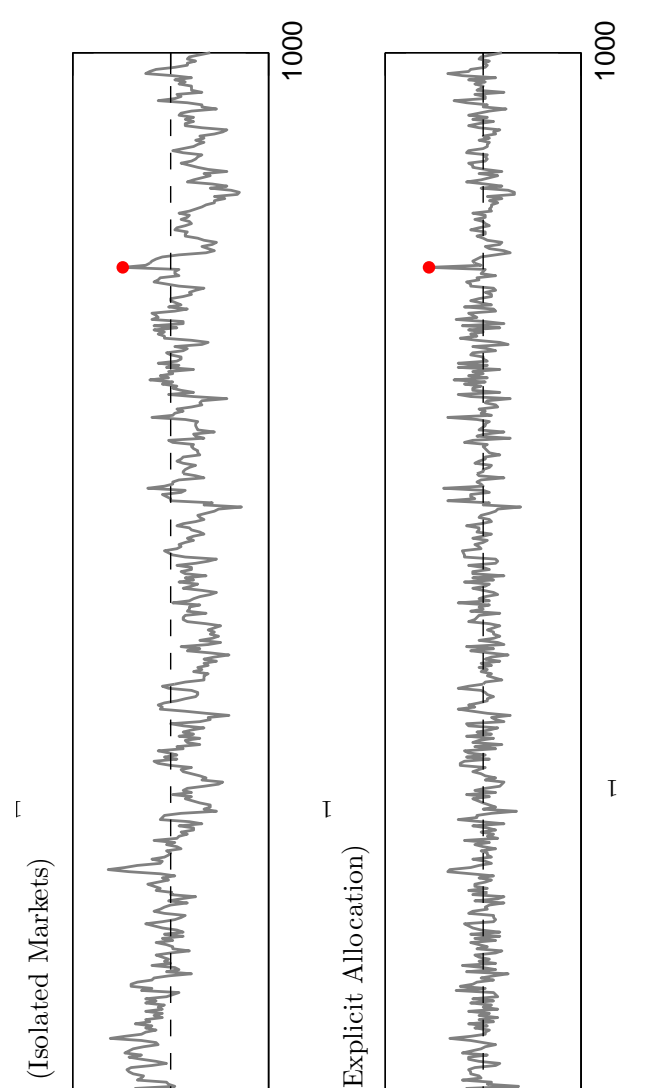

㩆琗

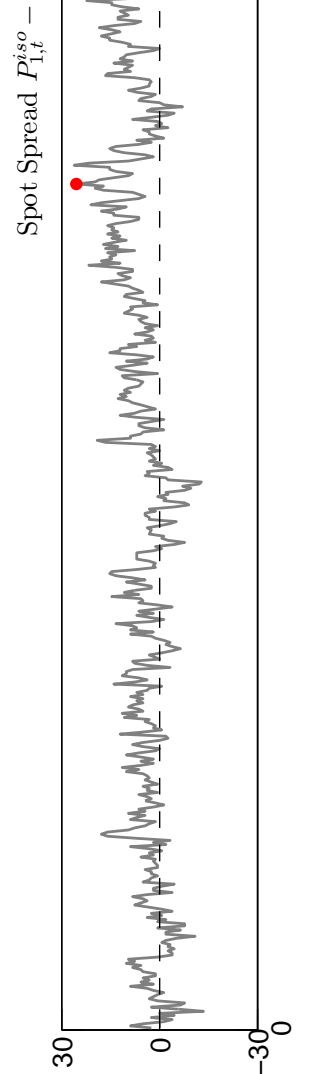

(чMN/YดA) peәлdS
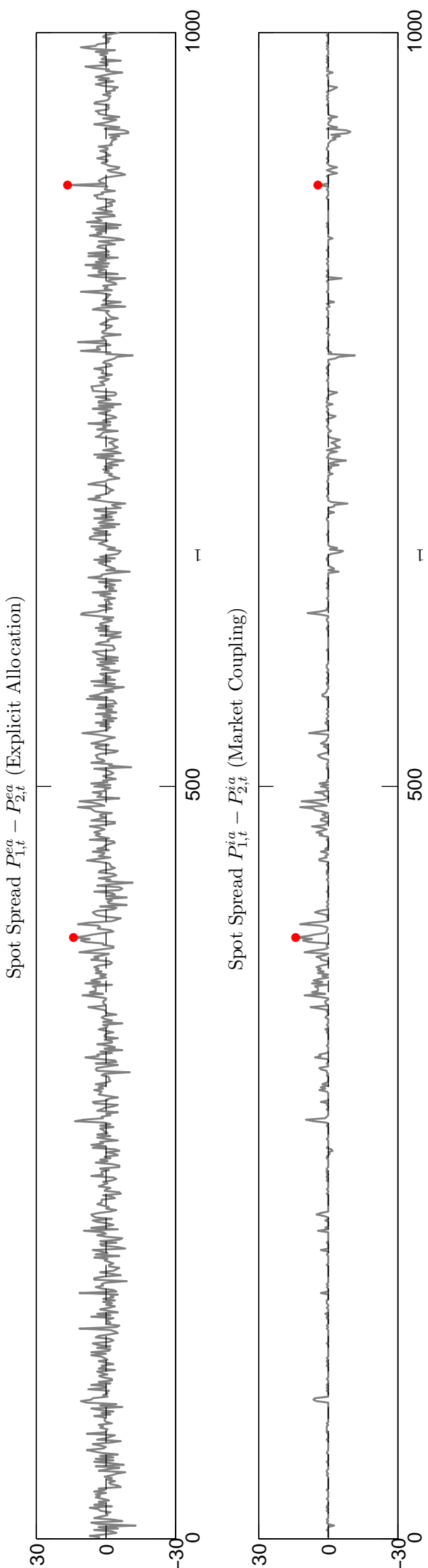

(чMN/4กษ) peə.IdS

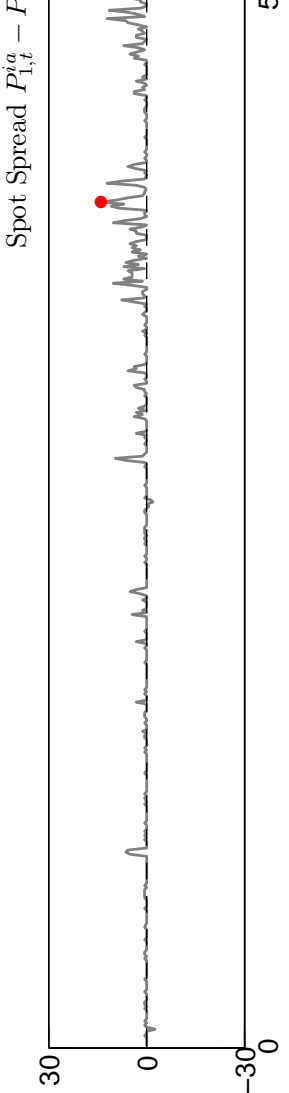

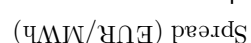

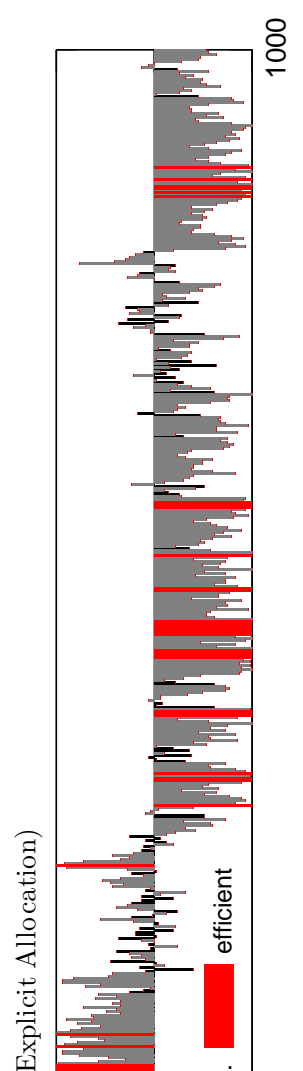

迎
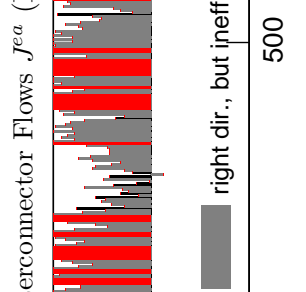

营

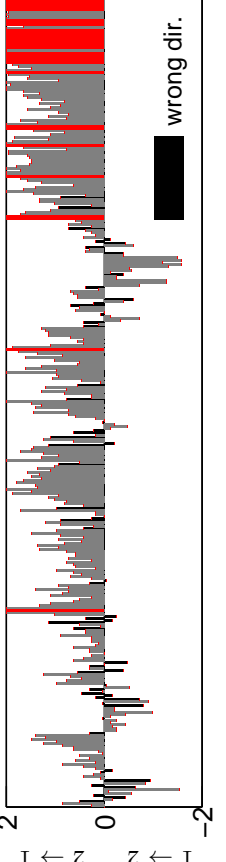

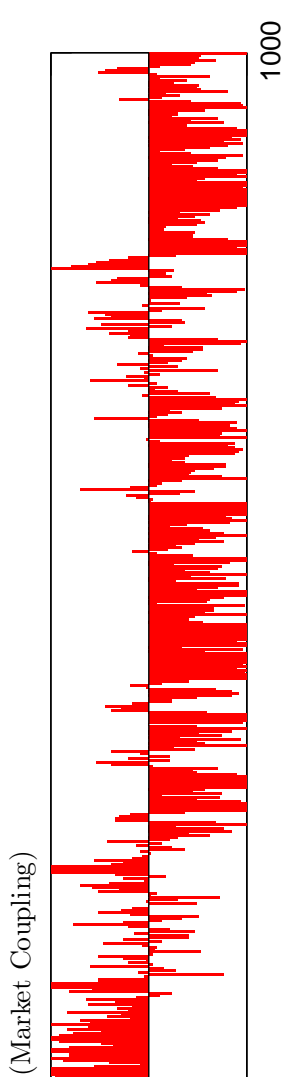
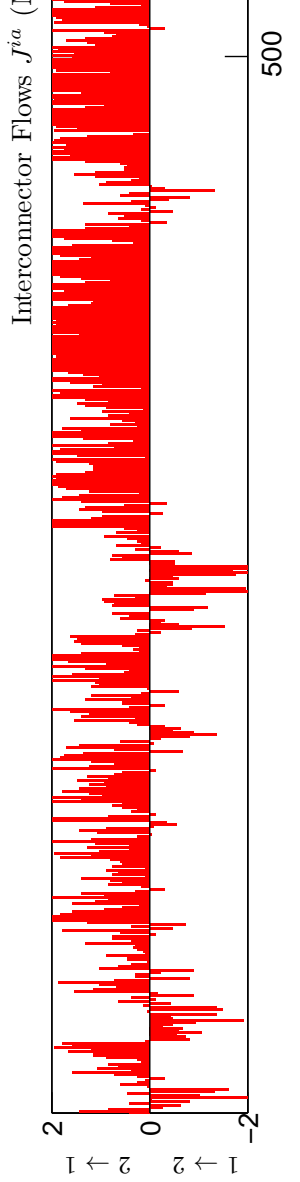

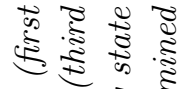

ग.

논

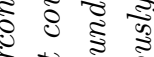

离 है

เ है

ह.

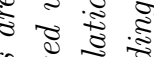

ग) हैं है

है के के

है है है

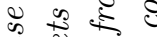

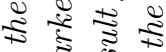

टे है क

๘ ईह क

क我事

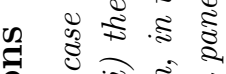

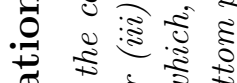

के ริ ₹

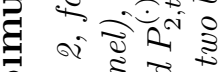

च छ ह छ छ

母)

की

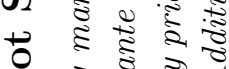

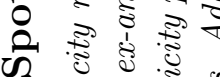

$\therefore$ i 3

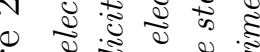

苟 ₹

T 0 \%

巡

吾

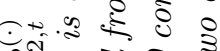

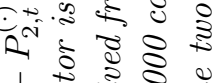

政

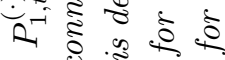

अ

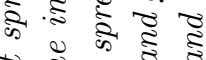

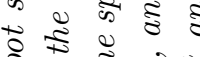
की

$\approx$

3.

का व 8 वे

हैँ : हो की

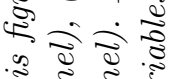

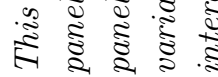



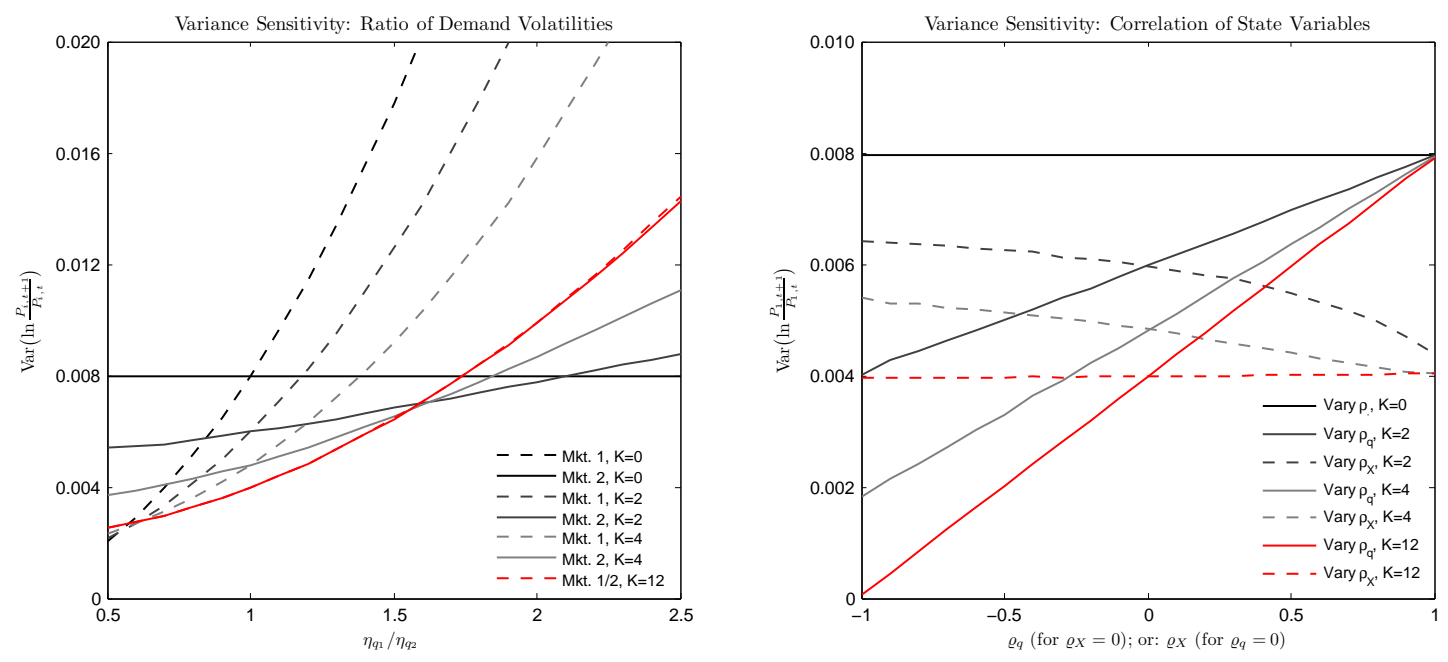

Figure 3: Sensitivities for Variance of log-Spot Returns

This figure shows sensitivities of the (unconditional) variance of log-spot returns $\ln \left(\frac{P_{i, t+1}}{P_{i, t}}\right)$ when varying key input parameters, and assuming given interconnector capacities $K$ of $0 G W$, 2GW, ${ }_{4} G W$, and $12 G W$, respectively. In the LHS graph, the variance of log-spot returns in both interconnected markets is plotted against the ratio of (instantaneous) volatilities, $\frac{\eta_{q_{1}}}{\eta_{q_{2}}}$, for the processes of electricity demand in both markets (as specified in Equation (2)). In the RHS graph, the variance for market 1 is analyzed when varying the correlation either between the processes for electricity demand or for the generating fuels in the two markets. 

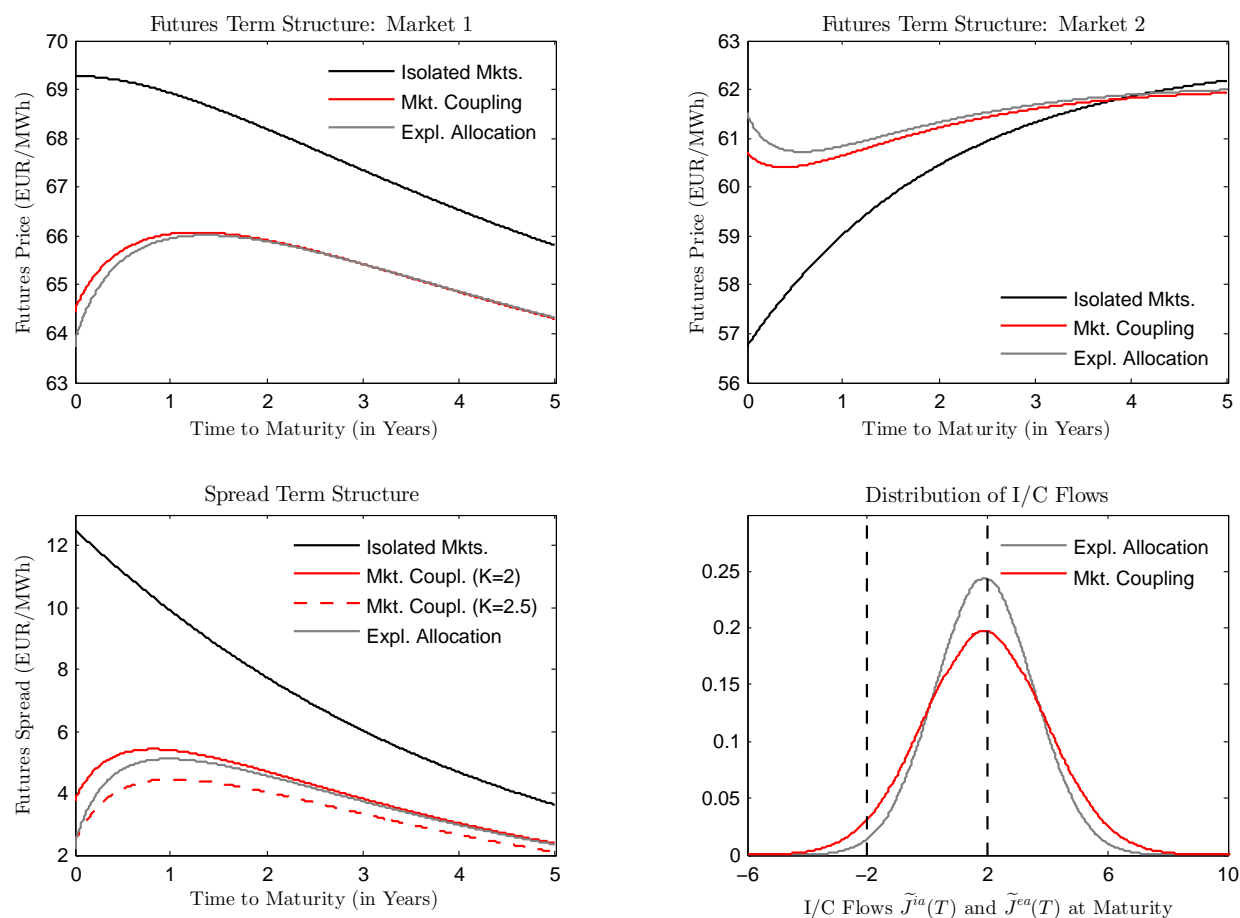

Figure 4: Futures and Futures Spread Term Structure

The top LHS and RHS graphs show the futures term structure in markets 1 and 2 for the cases that the capacity of the interconnector (i) is not available (isolated markets), (ii) is allocated via an explicit ex-ante scheme, or (iii) via an implicit scheme. The bottom LHS graph presents the corresponding spreads between futures prices in both markets. The bottom RHS graph displays the distribution of interconnector flows $\widetilde{J}^{\text {ea }}(T)$ and $\widetilde{J}^{i a}(T)$ for an assumed horizon of $T=30$ days. 